CERN-PH-TH/2012-341

\title{
On D-brane anti D-brane effective actions and their corrections to all orders in alpha-prime
}

\author{
Ehsan Hatefi 1 \\ International Centre for Theoretical Physics \\ Strada Costiera 11, Trieste, Italy \\ and \\ Theory Group, Physics Department, CERN \\ CH-1211, Geneva 23, Switzerland
}

\begin{abstract}
Based on a four point function, the S-matrix elements at disk level of the scattering amplitude of one closed string Ramond-Ramond field $(C)$, two tachyons and one scalar field, we find out new couplings in brane anti brane effective actions for $p=n, p+2=n$ cases. Using the infinite corrections of the vertex of one RR, one gauge and one scalar field and applying the correct expansion, it is investigated in detail how we produce the infinite gauge poles of the amplitude for $p=n$ case. By discovering new higher derivative corrections of two tachyontwo scalar couplings in brane anti brane systems to all orders in $\alpha^{\prime}$, we also obtain the infinite scalar poles in $\left(t^{\prime}+s^{\prime}+u\right)$-channel in field theory. Working with the complete form of the amplitude with the closed form of the expansion and comparing all the infinite contact terms of this amplitude, we derive several new Wess-Zumino couplings with all their infinite higher derivative corrections in the world volume of brane anti brane systems. In particular, in producing all the infinite scalar poles of $<V_{C} V_{\phi} V_{T} V_{T}>$, one has to consider the fact that scalar 's vertex operator in $(-1)$-picture must carry the internal $\sigma_{3}$ Chan-Paton matrix. The symmetric trace effective action has a non-zero coupling between $D \phi^{(1) i}$ and $D \phi_{i}^{(2)}$ while this coupling does not exist in ordinary trace effective action.
\end{abstract}

\footnotetext{
${ }^{1}$ E-mail:ehatefi@ictp.it,ehsan.hatefi@cern.ch
} 


\section{Introduction}

The relation between closed string Ramond-Ramond field and D-branes was widely realized in [1]. It is worthwhile to refer some of the main references on branes [2, 3, 4].

Concerning T-duality transformation, two important technical facts in the introduction of [5] have been addressed. However for good reasons we re-express the fact that for mix open-closed amplitudes (including closed string Ramond-Ramond) not all the coefficients of higher derivative corrections can be fixed in a correct manner, thus the direct calculations will be necessary ( as we clarify in detail this argument in section 3.3 of this paper).

To review within full details Dirac-Born-Infeld and Wess-Zumino effective actions [6, 7, 8, 9, 10] and all their references might be considered.

In this paper we would like to deal with non-BPS branes and in particular we look for new couplings (which can be explored just by S-matrix calculations) in the world volume of brane anti brane systems in type II super string theory, where spatial dimension of $D_{p}$-branes becomes odd (even) for IIA (IIB) string theory.

Studying tachyons in super string theories may provide some good information about these theories in some backgrounds which are time-dependent [11, 12, 13, 14, 15]. Based on some arguments [15], we highlight the point that the effective theory of all non-BPS branes does involve just massless states and tachyon. To be able to proceed with non-BPS branes in string theory within their applications, one should take into account [12, 13, 16]. The complete form of the effective actions of non-BPS branes was introduced in [17, 18].

The only consistent effective action for D-brane anti D-brane systems, based on direct S-matrix computations of one closed string Ramond-Ramond, two tachyons and one gauge field was appeared in [19]. In that paper we have shown that there was a non-zero coupling between $F^{(1)}$ and $F^{(2)}$ and we found all their infinite higher derivative corrections.

Note that for ordinary trace prescription, this coupling does not exist. It is important to emphasize that the effective action in [20] is not consistent with S-matrix computations and in fact symmetrized trace works out for super string computations. The reasons for such an argument ( see [19]) are as follows. By using the effective action appeared in [20], we are not able to produce all the tachyon poles of the amplitude of one RR, two tachyons and one gauge field, in addition to that if we use [20], the structures and some of the forms of new couplings (such as $F^{1} \cdot F^{2}$ which is confirmed by S-matrix in [19]) will be disappeared. 
In this paper we explore the presence of new term involving

$$
D \phi^{i(1)} \cdot D \phi_{i}^{(2)}
$$

in DBI action of brane anti brane systems where this new coupling and its all order $\alpha^{\prime}$ higher derivative corrections can be discovered just by applying S-matrix method of this paper. Having set the tachyon to zero, both ordinary and symmetric trace effective actions become equivalent.

In order to observe the details on tachyon condensation for brane anti brane system [21] has been constructed, moreover some of the the Ramond-Ramond couplings on the world volume of brane anti brane have been discussed within all needed details in [22, 19. Although several motivations including their explanations have been explained in [18, however, it is important to emphasize some of them very briefly and for the rest of motivations we just refer to all references that appeared in [18].

The first motivation is actually related to dualities [23]. The second one somehow should be taken as a matter of brane production in which we are not going to go through details so just to clarify more [24, 25] might be useful to look in. Indeed if the distance between brane anti brane is less than string's scale, then we will have two real tachyons in brane anti brane 's spectrum. The third motivation is to have inflation in string theory, one might consider brane anti brane effective action [19] in which brane and anti brane have to be separated [26, 27, 28, 29], in which this formalism was explained in detail in the section 2.1 of [18]. The fourth motivation for studying tachyons is to work out with holographic models in QCD [30, 31]. The last motivation is related to scattering amplitude method. Indeed the S-Matrix computation does have a very strong potential to explore several new couplings with having no on-shell ambiguity.

Having set some arguments [32, 22], one can talk about the Wess-Zumino effective action for the tachyons by applying super connection approach [33].

Note that the super connection's curvature just for brane anti brane has been achieved in [19] and it is generalized in [18] to actually take various couplings between gauge, tachyon and Ramond-Ramond field (RR) in the branes' world volume directions.

However the couplings between scalar field, RR and tachyons can not be derived by making use of the multiplication rule of the super matrices. The only way to discover these new couplings on the longitudinal and transverse directions of the brane anti brane is just based on scattering amplitude techniques. In [5, 6, 34] we found all the infinite corrections to BPS branes and in particular, special attention was paid to a conjecture on higher 
derivative corrections for both BPS and non-BPS branes in [35] where their applications to some dualities [36], Ads/CFT correspondence and in M-theory [37] were argued. Moreover in 38. we have analyzed in a type IIB flux compactification the transitions between two different vacua via D5/NS5 brane nucleation, more significantly some comparisons with KKLT and type IIA have been made.

In the next section we would like to compute in detail the amplitude of one RR, two tachyons and one scalar field in the world volume of brane anti brane systems. Then we move on to momentum expansion and discuss about a particular expansion.

We do have several goals for carrying out this computation. One of the important goals of this paper is to explicitly find out (by comparing the S-Matrix of $C \phi T T$ with field theory analysis) the presence of new terms including

$$
D \phi^{i(1)} \cdot D \phi_{i}^{(2)}
$$

in DBI action of brane anti brane systems where $\phi^{(1)}$ lives on brane and $\phi^{(2)}$ moves on anti brane or viceversa.

The second goal will be deriving (with explicit computations) the effective actions of brane anti brane systems and also discovering several new Wess-Zumino couplings with all their infinite $\alpha^{\prime}$ corrections. In section 3.1 we find all the infinite u-channel gauge poles with all infinite contact interactions for $p=n$ case in field theory. We find some new couplings like

$$
\partial_{i} C_{p-1} \wedge D T \wedge D T^{*}\left(\phi^{1}+\phi^{2}\right)^{i}
$$

we also obtain its infinite higher derivative corrections and fix its coefficient by making use of the $C \phi T T$ amplitude.

The third goal is as follows. We need to go on to achieve all infinite $\alpha^{\prime}$-higher derivative corrections of two tachyon, two scalar couplings of brane anti brane systems and in particular we clearly show that these new corrections of brane anti brane systems are completely different from non-BPS branes [18], more importantly their coefficients can not be read from higher derivative corrections of two tachyons and two gauge fields of brane anti brane systems [19] (detailed arguments will be highlighted in section 3.3 ). We explore these new two tachyon, two scalar couplings of brane anti brane systems and clearly check them by producing all infinite scalar poles of our string amplitude in $\left(u+s^{\prime}+t^{\prime}\right)$ - channel for $p+2=n$ case. For $p+2=n$ case we also obtain a new coupling like

$$
\epsilon^{a_{0} \cdots a_{p}} H_{i a_{0} \cdots a_{p}}\left(\phi^{(1)}-\phi^{(2)}\right)^{i} T T^{*}
$$


and fix its coefficient as well.

\section{The four point amplitude between one RR, one scalar field and two tachyons $(C \phi T T)$}

In order to find the infinite higher derivative corrections of two tachyon, two scalar couplings in the world volume of brane anti brane systems with exact coefficients, we have to have the complete form of the amplitude of $C \phi T T$. One might wonder whether two scalar two tachyon couplings of non-BPS branes [18] could be applied to brane anti brane systems. With in detail we will show that those corrections of non-BPS branes give rise inconsistent results at each order of $\alpha^{\prime}$ and in fact by using [18] we are not able to actually match neither first simple pole nor infinite scalar poles of the string amplitude of $C \phi T T$ in the world volume of brane anti brane systems with its field theory analysis.

In this paper within details we will point out that two scalar two tachyon couplings of brane anti brane systems to all orders are completely different from two tachyon two scalar couplings of non-BPS branes [18] and indeed we observe in a clear way that making use of those non-BPS couplings we get wrong result and we can not match all infinite scalar poles of the string theory amplitude.

With this remarkable motivation we start discovering in detail the S-matrix elements of CфTT.

The four point amplitude between one closed string RR, one gauge field and two tachyons $C A T T$ in detail in [19] has been done. In this paper we show that apparently one can read the general structure of higher derivative corrections of two scalars two tachyons from two tachyons and two gauge fields but there is a very important fact about corrections as follows.

All the coefficients of higher derivative corrections (just for mixed open-closed amplitude) can just be fixed by S-matrix method and not by any other method like T-duality transformation to the old computations (see footnote 1 of [34]) 2. Setting all physical state conditions we observe that there are various subtleties in the mixed open-closed tree level amplitudes where we will point out some of them later on.

Nevertheless, let us make some concrete arguments based on field theory analysis. Therefore before getting to the computations, we want to make some comments about apparent similarities between $C A T T$ and $C \phi T T$ amplitudes. The amplitude for $C \phi T T$ for

\footnotetext{
${ }^{2}$ We thank J.Polchinski and R.C.Myers for various discussions on this subject.
} 
$p=n$ case does include the infinite gauge poles and also for $p+2=n$ case, this amplitude does involve the infinite scalar poles. Notice that due to some kinematic constraints $C \phi T T$ does not have any tachyon pole while $C A T T$ in addition to infinite gauge poles has infinite tachyon poles for $p=n$ case.

The other fact which comes out from the direct computation is that for $C \phi T T$ amplitude neither in $p=n$ nor in $p+2=n$ case we do not have any double pole. This is important to highlight it such that after some computations we found a double pole for $p=n$ case in $C A T T$ amplitude. The other fact is that the S-matrix of $C \phi T T$ will make sense for $p=n, p+2=n$ cases while the amplitude of $C A T T$ was made of $p-2=n, p=n$ cases.

The last motivation for performing the calculations of $C \phi T T$ is as follows.

We could discover several new Wess-Zumino couplings meanwhile they are completely absent in CATT amplitude [19]. Therefore by some well known Conformal Field Theory methods in the world volume of brane anti brane systems ( in type II super string theory), we start finding S-matrix of two tachyons, one scalar field and one closed string RR field to indeed derive new couplings with all their higher derivative corrections to all orders of $\alpha^{\prime}$ where the idea of obtaining closed form of higher derivative corrections to all orders of $\alpha^{\prime}$ sounds interesting.

This $C \phi T T$ amplitude in brane anti brane system's world volume can be written down as follows

$$
\mathcal{A}^{C \phi T T} \sim \int d x_{1} d x_{2} d x_{3} d z d \bar{z}\left\langle V_{\phi}^{(0)}\left(x_{1}\right) V_{T}^{(0)}\left(x_{2}\right) V_{T}^{(0)}\left(x_{3}\right) V_{R R}^{\left(-\frac{3}{2},-\frac{1}{2}\right)}(z, \bar{z})\right\rangle .
$$

Note that we do have two more freedoms to find out the amplitude of $C \phi T T$, like working out either with

$$
\left\langle V_{\phi}^{(-1)}\left(x_{1}\right) V_{T}^{(0)}\left(x_{2}\right) V_{T}^{(0)}\left(x_{3}\right) V_{R R}^{\left(-\frac{1}{2},-\frac{1}{2}\right)}(z, \bar{z})\right\rangle
$$

or

$$
\left\langle V_{\phi}^{(0)}\left(x_{1}\right) V_{T}^{(-1)}\left(x_{2}\right) V_{T}^{(0)}\left(x_{3}\right) V_{R R}^{\left(-\frac{1}{2},-\frac{1}{2}\right)}(z, \bar{z})\right\rangle
$$

One remarkable fact about tachyon's vertex operator in string theory is that it does relate to two real components of a complex tachyon in field theory which means that the following relation holds

$$
T=\frac{1}{\sqrt{2}}\left(T_{1}+i T_{2}\right)
$$


In order to avoid some details we try to look for $C \phi T T$ amplitude in the following picture

$$
\mathcal{A}^{C \phi T T} \sim \int d x_{1} d x_{2} d x_{3} d z d \bar{z}\left\langle V_{\phi}^{(-1)}\left(x_{1}\right) V_{T}^{(0)}\left(x_{2}\right) V_{T}^{(0)}\left(x_{3}\right) V_{R R}^{\left(-\frac{1}{2},-\frac{1}{2}\right)}(z, \bar{z})\right\rangle,
$$

Indeed it is the fastest way to carry out $C \phi T T$ amplitude without making use of applying several physical state conditions and Bianchi identities. To clarify all things once more in this paper we express the general form of vertices for our amplitude 3

$$
\begin{aligned}
V_{T}^{(0)}(x) & =\alpha^{\prime} i k \cdot \psi(x) e^{\alpha^{\prime} i k \cdot X(x)} \lambda \otimes \sigma_{1} \\
V_{\phi}^{(-1)}(y) & =\xi_{i} e^{-\phi(y)} \psi^{i}(y) e^{\alpha^{\prime} i q X(y)} \lambda \otimes \sigma_{3} \\
V_{R R}^{(-1)}(z, \bar{z}) & =\left(P_{-} H_{(n)} M_{p}\right)^{\alpha \beta} e^{-\phi(z) / 2} S_{\alpha}(z) e^{i \frac{\alpha^{\prime}}{2} p \cdot X(z)} e^{-\phi(\bar{z}) / 2} S_{\beta}(\bar{z}) e^{i \frac{\alpha^{\prime}}{2} p \cdot D \cdot X(\bar{z})} \otimes \sigma_{3}
\end{aligned}
$$

Note that due to non-zero couplings between two tachyons and one RR in brane anti brane systems [19, 22] the Chan-Paton (CP) factor for RR for brane anti brane in (-1)picture (which is $\sigma_{3}$ ) is different from $\mathrm{CP}$ factor for $\mathrm{RR}$ in non-BPS branes which is $\sigma_{3} \sigma_{1}$.

$k$ is tachyon's momentum which satisfies $k^{2}=\frac{1}{4}$ and physical state conditions for scalar is $k_{2} \cdot \xi=k_{1} \cdot \xi=q . \xi=0$. The scalar in (-1)-picture does accompany $\sigma_{3}$ factor, the so called internal degree of freedom. Notice that the other CP factors of open strings and RR closed string for diverse pictures are introduced in [18]. Thus our amplitude has $\operatorname{Tr}\left(\sigma_{3} \sigma_{3} \sigma_{1} \sigma_{1}\right)=2$ factor. The projector in closed string is $P_{-}=\frac{1}{2}\left(1-\gamma^{11}\right)$ and

$$
H_{(n)}=\frac{a_{n}}{n !} H_{\mu_{1} \ldots \mu_{n}} \gamma^{\mu_{1}} \ldots \gamma^{\mu_{n}}
$$

with $n=2,4, a_{n}=i\left(n=1,3,5, a_{n}=1\right)$ for IIA (IIB). Having used doubling trick [18], we now replace fields to a total complex plane which means that the following change of variables have to be considered

$$
\tilde{X}^{\mu}(\bar{z}) \rightarrow D_{\nu}^{\mu} X^{\nu}(\bar{z}), \quad \tilde{\psi}^{\mu}(\bar{z}) \rightarrow D_{\nu}^{\mu} \psi^{\nu}(\bar{z}), \quad \tilde{\phi}(\bar{z}) \rightarrow \phi(\bar{z}), \quad \text { and } \quad \tilde{S}_{\alpha}(\bar{z}) \rightarrow M_{\alpha}{ }^{\beta} S_{\beta}(\bar{z})
$$

with

$$
D=\left(\begin{array}{cc}
-1_{9-p} & 0 \\
0 & 1_{p+1}
\end{array}\right), \quad \text { and } \quad M_{p}=\left\{\begin{array}{cc}
\frac{ \pm i}{(p+1) !} \gamma^{i_{1}} \gamma^{i_{2}} \ldots \gamma^{i_{p+1}} \epsilon_{i_{1} \ldots i_{p+1}} \text { for p even } \\
\frac{ \pm 1}{(p+1) !} \gamma^{i_{1}} \gamma^{i_{2}} \ldots \gamma^{i_{p+1}} \gamma_{11} \epsilon_{i_{1} \ldots i_{p+1}} \text { for } \mathrm{podd}
\end{array}\right.
$$

Once more, it is worth pointing out the needed correlators $X^{\mu}, \psi^{\nu}, \phi$, as below

$$
\begin{aligned}
\left\langle X^{\mu}(z) X^{\nu}(w)\right\rangle & =-\frac{\alpha^{\prime}}{2} \eta^{\mu \nu} \log (z-w), \\
\left\langle\psi^{\mu}(z) \psi^{\nu}(w)\right\rangle & =-\frac{\alpha^{\prime}}{2} \eta^{\mu \nu}(z-w)^{-1}, \\
\langle\phi(z) \phi(w)\rangle & =-\log (z-w) .
\end{aligned}
$$

\footnotetext{
${ }^{3}$ In string theory, one does set $\alpha^{\prime}=2$.
} 
By applying $x_{4} \equiv z=x+i y$ and $x_{5} \equiv \bar{z}=x-i y$, one can get the $C \phi T T$ amplitude as

$$
\begin{aligned}
\mathcal{A}^{C \phi T T} \sim & 2 \int d x_{1} d x_{2} d x_{3} d x_{4} d x_{5}\left(P_{-} H_{(n)} M_{p}\right)^{\alpha \beta} \xi_{1 i} k_{2 a}\left(-\alpha^{\prime 2} k_{3 b}\right) x_{45}^{-1 / 4}\left(x_{14} x_{15}\right)^{-1 / 2} I \\
& \times<: S_{\alpha}\left(x_{4}\right): S_{\beta}\left(x_{5}\right): \psi^{i}\left(x_{1}\right): \psi^{a}\left(x_{2}\right): \psi^{b}\left(x_{3}\right)>\operatorname{Tr}\left(\lambda_{1} \lambda_{2} \lambda_{3}\right),
\end{aligned}
$$

where

$$
I=\left|x_{12}\right|^{\alpha^{\prime 2} k_{1} \cdot k_{2}}\left|x_{13}\right|^{\alpha^{\prime 2} k_{1} \cdot k_{3}}\left|x_{14} x_{15}\right|^{\frac{\alpha^{\prime 2}}{2} k_{1} \cdot p}\left|x_{23}\right|^{\alpha^{\prime 2} k_{2} \cdot k_{3}}\left|x_{24} x_{25}\right|^{\frac{\alpha^{\prime 2}}{2} k_{2} \cdot p}\left|x_{34} x_{35}\right|^{\frac{\alpha^{\prime 2}}{2} k_{3} \cdot p}\left|x_{45}\right|^{\frac{\alpha^{\prime 2}}{4} p \cdot D \cdot p}
$$

such that $x_{i j}=x_{i}-x_{j}$ has been used. Applying the Wick-like rule [39] and [17, 6] to our case we end up with the correlation function involving three fermions $(\psi \mathrm{s})$ and two spin operators as

$$
\begin{aligned}
<: S_{\alpha}\left(x_{4}\right): S_{\beta}\left(x_{5}\right): \psi^{i}\left(x_{1}\right): \psi^{a}\left(x_{2}\right):: \psi^{b}\left(x_{3}\right):>= & {\left[\left(\Gamma^{b a i} C^{-1}\right)_{\alpha \beta}-2 \eta^{a b} \frac{\operatorname{Re}\left[x_{24} x_{35}\right]}{x_{23} x_{45}}\left(\gamma^{i} C^{-1}\right)_{\alpha \beta}\right] } \\
& \times 2^{-3 / 2} x_{45}^{1 / 4}\left(x_{14} x_{15} x_{24} x_{25} x_{34} x_{35}\right)^{-1 / 2}
\end{aligned}
$$

Embedding (6) into our amplitude, we were able to check the $\operatorname{SL}(2, \mathrm{R})$ invariance of the S-matrix of $C \phi T T$. We wish to fix the location of the open strings in special places, that is

$$
x_{1}=0, \quad x_{2}=1, \quad x_{3} \rightarrow \infty
$$

By using this particular gauge fixing, we get to the following integrals

$$
\int d^{2} z|1-z|^{a}|z|^{b}(z-\bar{z})^{c}(z+\bar{z})^{d}
$$

where $d=0,1,2$ and $a, b, c$ should be given in terms of the Mandelstam variables as below

$$
s=-\frac{\alpha^{\prime}}{2}\left(k_{1}+k_{3}\right)^{2}, \quad t=-\frac{\alpha^{\prime}}{2}\left(k_{1}+k_{2}\right)^{2}, \quad u=-\frac{\alpha^{\prime}}{2}\left(k_{2}+k_{3}\right)^{2} .
$$

The result of the integrations for just $d=0,1$ was expressed in [40], however if the computations were done in $C^{-2} \phi^{0} T^{0} T^{0}$ picture we would need the result of the integrations for $d=2$ as well, which was performed in [18].

After some computations now we write down the complete form of $C \phi T T$,

$$
\mathcal{A}^{C \phi T T}=\mathcal{A}_{1}+\mathcal{A}_{2}
$$


so that

$$
\begin{aligned}
& \mathcal{A}_{1} \sim-8 \xi_{1 i} k_{2 a} k_{3 b} 2^{-3 / 2} \operatorname{Tr}\left(P_{-} H_{(n)} M_{p} \Gamma^{b a i}\right) L_{1}, \\
& \mathcal{A}_{2} \sim-8 \xi_{1 i} 2^{-3 / 2}\left\{\operatorname{Tr}\left(P_{-} H_{(n)} M_{p} \gamma^{i}\right)\right\} L_{2}
\end{aligned}
$$

It should be emphasized that the closed form of $<V_{C} V_{\phi} V_{T} V_{T}>$ does not vanish only for $p=n$ and $p+2=n$ cases. As a matter of fact we observe that $\mathcal{A}_{1}$ (first part of amplitude) in (9) is antisymmetric under interchanging two tachyons or under replacing $2 \leftrightarrow 3$. This does show that the first part of this four point function for $p=n$ case should be vanished for both one RR and two real $T_{1}$ tachyons and one RR and two $T_{2}$ 's .

$L_{1}, L_{2}$ are made of

$$
\begin{aligned}
& L_{1}=(2)^{-2(t+s+u)-1} \pi \frac{\Gamma(-u) \Gamma\left(-s+\frac{1}{4}\right) \Gamma\left(-t+\frac{1}{4}\right) \Gamma(-t-s-u)}{\Gamma\left(-u-t+\frac{1}{4}\right) \Gamma\left(-t-s+\frac{1}{2}\right) \Gamma\left(-s-u+\frac{1}{4}\right)}, \\
& L_{2}=-(2)^{-2(t+s+u+1)} \pi \frac{\Gamma\left(-u+\frac{1}{2}\right) \Gamma\left(-s+\frac{3}{4}\right) \Gamma\left(-t+\frac{3}{4}\right) \Gamma\left(-t-s-u-\frac{1}{2}\right)}{\Gamma\left(-u-t+\frac{1}{4}\right) \Gamma\left(-t-s+\frac{1}{2}\right) \Gamma\left(-s-u+\frac{1}{4}\right)}
\end{aligned}
$$

By talking about the correct momentum expansion we will actually understand that how to gain all infinite scalar, gauge poles for different $p, n$ values, how to interpret them in field theory and how to look for new couplings in the world volume of brane anti brane systems. We also explain why we do not have any tachyon pole in $<V_{C} V_{\phi} V_{T} V_{T}>$.

\section{Momentum expansion for brane-anti brane systems}

To be able to look for all couplings in string theory, we need to expand the complete form of the S-matrix elements to find all singular terms (poles) and contact interactions. However due to presence of tachyons here the expansion is no longer the low energy expansion. Therefore we are not allowed to use the limit of $\alpha^{\prime} \rightarrow 0$ of the above string amplitude. Turning into Mandelstam variables and using the momentum conservation along the world volume of brane, $\alpha^{\prime}\left(k_{1}^{a}+k_{2}^{a}+k_{3}^{a}\right)+p^{a}+(p . D)^{a}=0$, we obtain a very useful constraint as follows

$$
s+t+u=-p_{a} p^{a}-\frac{1}{2}
$$

In [18] we discussed in detail that the momentum expansion for various amplitudes should be found either by $\left(k_{i}+k_{j}\right)^{2} \rightarrow 0$ or $k_{i} \cdot k_{j} \rightarrow 0$. The first case takes place when we 
do have a massless pole and for the other cases we should use $k_{i} \cdot k_{j} \rightarrow 0$ expansion. Due to non-zero two tachyon one gauge couplings, we realize that our amplitude $C \phi T T$ has to have a massless pole in $-\left(k_{3}+k_{2}\right)^{2}=u$ channel. It should only have infinite scalar poles in $t^{\prime}+s^{\prime}+u$ channel $\left(t^{\prime}=t+\frac{1}{4}, s^{\prime}=s+\frac{1}{4}\right)$ and it will become clear later on.

The extremely important point for brane -anti brane systems is that the quantity $p^{a} p_{a}$ should tend to zero while in non-BPS systems due to kinematic constraints (which Mandelstam variables justify) our amplitude like $C \phi \phi T\left[18\right.$ just makes sense for $p^{a} p_{a} \rightarrow \frac{1}{4}$.

Keeping in mind above arguments, we understand that the correct momentum expansion in brane anti brane system is indeed

$$
\left(k_{3}+k_{2}\right)^{2} \rightarrow 0, \quad k_{1} \cdot k_{3} \rightarrow 0, \quad k_{2} \cdot k_{1} \rightarrow 0 .
$$

Also using the on-shell relations $k_{1}^{2}=0$ and $k_{2}^{2}=k_{3}^{2}=\frac{1}{4}$ the above momentum expansion can be interpreted in terms of Mandelstam variables as

$$
u \rightarrow 0, \quad s \rightarrow \frac{-1}{4}, \quad t \rightarrow \frac{-1}{4}
$$

Therefore $C \phi T T$ should be performed just by setting $p_{a} p^{a} \rightarrow 0$ in brane-anti brane systems.

Applying (11) into the general form of the amplitude, we conclude the poles of the Gamma functions. Hence our amplitude for $p=n$ case, has infinite $\mathrm{u}$-channel gauge poles meanwhile it does have infinite scalar poles for $p+2=n$ case. Note that since the two tachyons, one scalar field coupling is vanished, we no longer have tachyon pole, rather than in $C A T T$ amplitude for $p=n$ case we had infinite tachyon poles.

Now we would like to expand our amplitude around (11) to find new couplings out and also to gain the correct two tachyon two scalar couplings of brane anti brane systems to all orders in $\alpha^{\prime}$. We also do want to confirm all order corrections to the Wess-Zumino effective actions of BPS branes which have recently been constructed in [34, 35]. By deriving the closed form of the expansions, we search about several new couplings in field theory, set their coefficients precisely and eventually find all their infinite higher derivative corrections thereof. 


\subsection{Infinite u-channel gauge poles and contact interactions for $p=n$ case}

The amplitude was anti symmetrized with respect to interchanging two external tachyons therefore both four point amplitudes of $C \phi T_{1} T_{1}$ and $C \phi T_{2} T_{2}$ make no sense in this case. By performing the trace, the ultimate form of the amplitude of $C \phi T_{1} T_{2}$ becomes

$$
\mathcal{A}^{C \phi T_{1} T_{2}}=-8 \xi_{1 i} k_{3 b} k_{2 a} 2^{-3 / 2} \frac{16}{(p) !}\left(\epsilon^{a_{0} \cdots a_{p-2} b a} H_{a_{0} \cdots a_{p-2}}^{i}\right) L_{1}
$$

Let us include the expansion of $L_{1}$ around (11) at leading orders

$$
L_{1}=\pi^{3 / 2}\left(\frac{-1}{u}+4 \ln (2)+\left(\frac{\pi^{2}}{6}-8 \ln (2)^{2}\right) u-\frac{\pi^{2}}{6} \frac{\left(s^{\prime}+t^{\prime}\right)^{2}}{u}+\cdots\right)
$$

such that $s^{\prime}=s+\frac{1}{4}, t^{\prime}=t+\frac{1}{4}$.

It is clear from (13) that the first term is pole, thus we have to produce this simple pole in field theory as well, although as we will see later this part of the S-matrix involves infinite gauge poles, however for the moment let us proceed order by order. In order to produce that gauge pole, we should have taken the following Feynman rule :

$$
\mathcal{A}=V_{a}\left(C_{p-1}, \phi, A\right) G_{a b}(A) V_{b}\left(A, T_{1}, T_{2}\right)
$$

with

$$
\begin{aligned}
G_{a b}(A) & =\frac{i \delta_{a b}}{\left(2 \pi \alpha^{\prime}\right)^{2} T_{p} k^{2}} \\
V_{b}\left(A, T_{1}, T_{2}\right) & =T_{p}\left(2 \pi \alpha^{\prime}\right)\left(k_{2 b}-k_{3 b}\right)
\end{aligned}
$$

Note that in the propagator $k^{2}$ is indeed $-\frac{\alpha^{\prime}}{2}\left(k_{2}+k_{3}\right)^{2}=u$. To make field theory obvious, we just point out that the so called $\operatorname{Tr}\left(\frac{-1}{4} F_{a b} F^{b a}\right)$ (gauge field's kinetic term) is incharge of propagator.

$V_{b}\left(A, T_{1}, T_{2}\right)$ has been found from $\operatorname{Tr}\left(2 \pi \alpha^{\prime} D_{a} T D^{a} T\right)$ (tachyon's kinetic term), by applying direct field theory techniques.

In order to obtain that pole we need to actually have the coupling of $V_{a}\left(C_{p-1}, \phi, A\right)$ as follows:

$$
\mu_{p} \lambda\left(2 \pi \alpha^{\prime}\right) \int_{\sum_{(p+1)}} \operatorname{Tr}\left(\partial_{i} C_{p-1} \wedge F\left(\phi_{1}+\phi_{2}\right)^{i}\right)
$$


where $\lambda=2 \pi \alpha^{\prime}$ and we used the fact that amplitude is antisymmetric. The scalar field was found from Taylor expansion. Note that this coupling without $\phi_{2}^{i}$ has been firstly pointed out in 34 and it was obtained by carrying out direct computation of $C \phi A A$ amplitude in the world volume of BPS branes. Having set (16), we get to

$$
V_{a}\left(C_{p-1}, \phi, A\right)=\mu_{p}\left(2 \pi \alpha^{\prime}\right)^{2} \frac{1}{(p) !} \epsilon_{a_{0} \cdots a_{p-2}} H^{i a_{0} \cdots a_{p-2}} k_{a} \xi_{i}
$$

with $k$ becomes the off-shell gauge field's momentum and it is $k_{a}=-\left(k_{2}+k_{3}\right)_{a}$. It is not difficult to indeed show that by normalizing the amplitude with a coefficient of $\frac{i \mu_{p}}{4 \sqrt{2 \pi}}$ and using (14) the first gauge pole in (13) will precisely be resulted.

Now we come to interesting point which is discovering new couplings. As it is clear from the expansion, the second term in (13) is contact term so in order to produce this term the following coupling should have been considered

$$
\frac{i}{2} \mu_{p} \beta^{2}\left(2 \pi \alpha^{\prime}\right)^{3} \operatorname{Tr}\left(\partial_{i} C_{p-1} \wedge D T \wedge D T^{*}\left(\phi^{1}+\phi^{2}\right)^{i}\right)
$$

Thus by extracting this new Wess-Zumino coupling as follows

$$
A_{c}=\mu_{p} \beta^{2}\left(2 \pi \alpha^{\prime}\right)^{3} \frac{i}{2 p !} \epsilon_{a_{0} \cdots a_{p}} H^{i a_{0} \cdots a_{p-2}} k^{3 a_{p-1}} k^{2 a_{p}} \xi^{i}
$$

we could exactly produce the second term of the expansion. Notice that $\beta$ is a normalization constant in Wess-Zumino terms which has been fixed in [19].

Again the third term of the expansion is contact interaction. A very important question which comes out is that how we can talk about the other terms appeared in (13). The simple expectation is that the other terms must be related to higher derivative corrections of the new couplings so having applied this remarkable argument, we are led to the following coupling

$$
\frac{i}{2} \mu_{p}\left(2 \pi \alpha^{\prime}\right)\left(\alpha^{\prime}\right)^{2}\left(\frac{\pi^{2}}{6}-8 \ln 2^{2}\right) \operatorname{Tr}\left(\partial_{i} C_{p-1} \wedge D^{a} D_{a}\left(D T \wedge D T^{*}\right)\left(\phi^{1}+\phi^{2}\right)^{i}\right)
$$

Having used the higher derivative correction to the coupling of

$$
\operatorname{Tr}\left(\partial_{i} C_{p-1} \wedge D T \wedge D T^{*}\left(\phi^{1}+\phi^{2}\right)^{i}\right)
$$


as appeared in (19), we could produce the third term of the expansion in (13) in a correct manner, so not only could we find new coupling like (17) but also we could fix its coefficient, more interestingly we could obtain its higher derivative extensions in (19) without any on-shell ambiguity.

The other fascinating fact which we must emphasize is that, the expansion (13) dictates us that our S-matrix has namely infinitely many gauge poles. For example the fourth term in the expansion of (13) is again simple pole. All the infinite gauge poles are going to be produced by the same Feynman rule (14) but two subtleties are in order. First of all the whole infinite gauge poles will show us that the term of $\operatorname{Tr}\left(2 \pi \alpha^{\prime} D_{a} T D^{a} T\right)$ or in the other words, tachyon's kinetic term does not receive any higher derivative correction. This is related to the fact that this kinetic term or $\operatorname{Tr}\left(2 \pi \alpha^{\prime} D_{a} T D^{a} T\right)$ has been fixed in tachyon effective action. Second of all, the natural point comes out is that, the only approach to obtain all infinite gauge poles is to devote the higher derivative corrections to the vertex of $V_{a}\left(C_{p-1}, A, \phi^{i}\right)$. Thus it must get improved to be able to produce all infinite poles in field theory analysis as well.

For example the second pole for this amplitude is produced by embedding the following vertex in the Feynman amplitude (14)

$$
V_{a}\left(C_{p-1}, \phi, A\right)=\frac{\left(\alpha^{\prime} \pi\right)^{2}}{6} \mu_{p}\left(2 \pi \alpha^{\prime}\right)^{2} \frac{1}{(p) !} \epsilon_{a_{0} \cdots a_{p-2}} H^{i a_{0} \cdots a_{p-2}} k_{a} \xi_{i}\left(k_{1} \cdot p\right)^{2}
$$

Now in order to produce all infinite gauge poles, the complete form of the expansion of $L_{1}$ is needed. After applying some identities, the closed form of the expansion of $L_{1}$ around (11) to all orders in $\alpha^{\prime}$ can be explored as follows

$$
L_{1}=\pi^{3 / 2}\left(-\frac{1}{u} \sum_{n=-1}^{\infty} b_{n}\left(s^{\prime}+t^{\prime}\right)^{n+1}+\sum_{p, n, m=0}^{\infty} f_{p, n, m} u^{p}\left(s^{\prime} t^{\prime}\right)^{n}\left(s^{\prime}+t^{\prime}\right)^{m}\right)
$$

with $f_{p, 0,0}=a_{p}$ and the following coefficients must be considered

$$
\begin{aligned}
& b_{-1}=1, b_{0}=0, b_{1}=\frac{\pi^{2}}{6}, b_{2}=2 \zeta(3), a_{0}=4 \ln 2, \\
& a_{1}=\frac{\pi^{2}}{6}-8 \ln (2)^{2}, a_{2}=\frac{2}{3}\left(-\pi^{2} \ln 2+3 \zeta(3)+16 \ln (2)^{3}\right), \\
& f_{0,0,2}=\frac{2}{3} \pi^{2} \ln (2), f_{0,1,0}=-14 \zeta(3), f_{0,0,3}=8 \zeta(3) \ln (2), \\
& f_{1,1,0}=56 \zeta(3) \ln (2)-1 / 2, f_{1,0,2}=\frac{1}{36}\left(\pi^{4}-48 \pi^{2} \ln (2)^{2}\right), f_{0,1,1}=-1 / 2
\end{aligned}
$$


Note that some of these coefficients are completely different from those appeared in the world volume of non-BPS branes [18]. However it is interesting that $b_{n}$ coefficients of this paper are precisely the same $b_{n}$ 's that were appeared in the momentum expansion of BPS branes ( just like $b_{n}$ coefficients in the S-matrix elements of $C A A A$ in [6]).

By imposing the infinite higher derivative corrections as

$$
\mu_{p} \lambda\left(2 \pi \alpha^{\prime}\right) \int_{\sum_{(p+1)}} \partial_{i} C_{p-1} \wedge \sum_{n=-1}^{\infty} b_{n}\left(\alpha^{\prime}\right)^{n+1} \operatorname{Tr}\left(D_{a_{1}} \cdots D_{a_{n+1}} F D^{a_{1}} \cdots D^{a_{n+1}}\left(\phi_{1}+\phi_{2}\right)^{i}\right)
$$

deriving the vertex of $V_{a}\left(C_{p-1}, \phi, A\right)$ as

$$
V_{a}\left(C_{p-1}, \phi, A\right)=\mu_{p}\left(2 \pi \alpha^{\prime}\right)^{2} \frac{1}{(p) !} \epsilon_{a_{0} \cdots a_{p-2}} H^{i a_{0} \cdots a_{p-2}}\left(k_{2}+k_{3}\right)^{a} \xi_{i} \sum_{n=-1}^{\infty} b_{n}\left(\alpha^{\prime} k_{1} \cdot k\right)^{n+1}
$$

and replacing it in (14) we end up with

$$
\mathcal{A}=\mu_{p}\left(2 \pi \alpha^{\prime}\right) \frac{2 i}{(p) ! u} \epsilon_{a_{0} \cdots a_{p-2} a b} H^{i a_{0} \cdots a_{p-2}} k_{2}^{b} k_{3}^{a} \xi_{i} \sum_{n=-1}^{\infty} b_{n}\left(\frac{\alpha^{\prime}}{2}\right)^{n+1}\left(s^{\prime}+t^{\prime}\right)^{n+1}
$$

By replacing the first term of (20) in (12) and comparing (23) with (12) we actually clarified that the infinite string theory 'gauge poles are precisely produced. The other remarkable fact which should be emphasized is that,by comparing the above amplitude (12) with the infinite gauge poles of the field theory amplitude, that is, (23) we have no longer any residual contact interactions for $p=n$ case.

Let us end this part of the amplitude by searching about all contact terms of $C \phi T T$ amplitude as below

$$
-\frac{i}{2} \mu_{p} \pi \xi_{1 i} k_{3 b} k_{2 a} \frac{16}{(p) !}\left(\epsilon^{a_{0} \cdots a_{p-2} b a} H_{a_{0} \cdots a_{p-2}}^{i}\right) \sum_{p, n, m=0}^{\infty} f_{p, n, m} u^{p}\left(s^{\prime} t^{\prime}\right)^{n}\left(s^{\prime}+t^{\prime}\right)^{m}
$$

Working out in detail we can show that the closed form of all infinite contact terms of string theory amplitude for $p=n$ case, will be concluded in the field theory by taking into account the following couplings to all orders in $\alpha^{\prime}$ :

$$
\begin{aligned}
& 2 i \alpha^{\prime}\left(\pi \alpha^{\prime}\right) \mu_{p} \sum_{p, n, m=0}^{\infty} f_{p, n, m}\left(\frac{\alpha^{\prime}}{2}\right)^{p}\left(\alpha^{\prime}\right)^{2 n+m} \partial_{i} C_{p-1} \wedge\left(D^{a} D_{a}\right)^{p} D_{b_{1}} \cdots D_{b_{m}}\left(D_{a_{1}} \cdots D_{a_{n}} D T\right. \\
& \left.\wedge D_{a_{n+1}} \cdots D_{a_{2 n}} D T^{*}\right) \partial^{a_{1}} \cdots \partial^{a_{2 n}} \partial^{b_{1}} \cdots \partial^{b_{m}}\left(\phi_{1}+\phi_{2}\right)^{i}
\end{aligned}
$$


Note that in the above coupling we could consider covariant derivative of scalar, however it turns out that the commutator in the definition of covariant derivative of scalar should not be considered here as we are looking for $C \phi T T$ coupling. A good question is to see whether or not covariant derivative of scalar keeps fixed in the above coupling. To answer this question one has to carry out a six point function, basically checking $C \phi A T T$ amplitude will be worth, nevertheless we can not come over to this remark by our computations of this paper.

\subsection{Infinite $\left(u+s^{\prime}+t^{\prime}\right)$-channel scalar poles and contact interac- tions for $p+2=n$ case}

Having performed the trace, we can take all non-zero terms of the string amplitude for this certain case as

$$
\mathcal{A}^{\phi T_{1} T_{1} C}= \pm \frac{8 i \mu_{p}}{\sqrt{\pi}(p+1) !} L_{2} \epsilon^{a_{0} \cdots a_{p}} H_{a_{0} \cdots a_{p}}^{i} \xi_{i}
$$

As it follows from the amplitude and unlike the first part of the amplitude, in this section our S-matrix is symmetric, that is, by interchanging two tachyons the amplitude remains unchanged. Therefore $C \phi T_{1} T_{2}$ amplitude does not have any contribution to $p+2=n$ case. As a matter of fact our calculations make sense either for $C \phi T_{1} T_{1}$ or $C \phi T_{2} T_{2}$ amplitudes. Note that due to kinematic reasons, we can not talk about associated ward identities either.

The expansion of $L_{2}$ at leading order around (11) is

$$
\begin{aligned}
L_{2}= & \frac{\sqrt{\pi}}{2}\left(\frac{-1}{\left(t^{\prime}+s^{\prime}+u\right)}+4 \ln (2)+\left(\frac{\pi^{2}}{6}-8 \ln (2)^{2}\right)\left(s^{\prime}+t^{\prime}+u\right)\right. \\
& \left.-\frac{\pi^{2}}{3} \frac{t^{\prime} s^{\prime}}{\left(t^{\prime}+s^{\prime}+u\right)}+\cdots\right)
\end{aligned}
$$

The first scalar pole in $t^{\prime}+s^{\prime}+u$-channel should be produced by taking the following amplitude

$$
\begin{aligned}
\mathcal{A}= & V_{i}\left(C_{p+1}, \phi^{(1)}\right) G_{i j}(\phi) V_{j}\left(\phi^{(1)}, T_{1}, T_{1}, \phi^{(1)}\right) \\
& +V_{i}\left(C_{p+1}, \phi^{(2)}\right) G_{i j}(\phi) V_{j}\left(\phi^{(2)}, T_{1}, T_{1}, \phi^{(1)}\right)
\end{aligned}
$$

It is important to highlight the fact that in order to have consistent result $\phi$ in the propagator must be $\phi^{(1)}$ and $\phi^{(2)}$. The needed vertices can be read off as 


$$
\begin{aligned}
G_{i j}(\phi) & =\frac{i \delta_{i j} \delta_{\alpha \beta}}{\left(2 \pi \alpha^{\prime}\right)^{2} T_{p}\left(u+t^{\prime}+s^{\prime}\right)} \\
V_{i}\left(C_{p+1}, \phi^{(1)}\right) & =i \mu_{p}\left(2 \pi \alpha^{\prime}\right) \frac{1}{(p+1) !} \epsilon^{a_{0} \cdots a_{p}} H_{i a_{0} \cdots a_{p}} \operatorname{Tr}\left(\lambda_{\alpha}\right) \\
V_{i}\left(C_{p+1}, \phi^{(2)}\right) & =-i \mu_{p}\left(2 \pi \alpha^{\prime}\right) \frac{1}{(p+1) !} \epsilon^{a_{0} \cdots a_{p}} H_{i a_{0} \cdots a_{p}} \operatorname{Tr}\left(\lambda_{\alpha}\right) \\
V_{j}\left(\phi^{(1)}, T_{1}, T_{1}, \phi^{(1)}\right) & =-2 i T_{p}\left(2 \pi \alpha^{\prime}\right) \xi_{j} \operatorname{Tr}\left(\lambda_{1} \lambda_{2} \lambda_{3} \lambda_{\beta}\right) \\
V_{j}\left(\phi^{(2)}, T_{1}, T_{1}, \phi^{(1)}\right) & =2 i T_{p}\left(2 \pi \alpha^{\prime}\right) \operatorname{Tr}\left(\lambda_{1} \lambda_{2} \lambda_{3} \lambda_{\beta}\right) \xi_{j}
\end{aligned}
$$

Now by applying the above vertices into (28) we reach to

$$
\mathcal{A}=\frac{4 i \mu_{p}}{(p+1) !\left(u+s^{\prime}+t^{\prime}\right)} \epsilon^{a_{0} \cdots a_{p}} H_{i a_{0} \cdots a_{p}} \xi^{i}
$$

By substituting (27) into (28) and comparing the result with (30) we get the fact that the first scalar pole in $u+t^{\prime}+s^{\prime}$-channel has been precisely obtained.

From this part of the amplitude we understand that the presence of

$$
D \phi^{(1) i} \cdot D \phi_{i}^{(2)}
$$

term in the effective action is inevitable. In fact if we do not consider $D \phi^{(1) i} \cdot D \phi_{i}^{(2)}$ term in the effective action then $V_{j}\left(\phi^{(2)}, T_{1}, T_{1}, \phi^{(1)}\right)$ does not have any value and naturally we are not able to produce even the first simple scalar pole in field theory where this new term (31) is not inside of ordinary trace effective action.

Let us consider the first contact interaction in (27) as follows

$$
\mathcal{A}_{c}=i \mu_{p} \ln (2) \frac{16}{(p+1) !} \epsilon^{a_{0} \cdots a_{p}} H_{i a_{0} \cdots a_{p}} \xi^{i}
$$

It is remarkable to point out the following point. In order to produce (32), we should explore another new coupling and indeed fix its coefficient, which is the only way to do so as

$$
\frac{\mu_{p}}{(p+1) !}\left(2 \pi \alpha^{\prime}\right)^{2} \beta^{2} \partial_{i} C_{a_{0} \cdots a_{p}}\left(\phi^{(1)}-\phi^{(2)}\right)^{i}|T|^{2} \epsilon^{a_{0} \cdots a_{p}}
$$


We believe that the third term in the expansion of (27), will be produced just by applying higher derivative on the above coupling as

$$
-\left(\alpha^{\prime}\right)^{2} \frac{\mu_{p}}{(p+1) !}\left(\frac{\pi^{2}}{6}-8 \ln (2)^{2}\right) \partial_{i} C_{a_{0} \cdots a_{p}} D^{a} D_{a}\left[\left(\phi^{(1)}-\phi^{(2)}\right)^{i}|T|^{2}\right] \epsilon^{a_{0} \cdots a_{p}}
$$

Another way of writing this higher extension is as follows :

$$
-\left(\alpha^{\prime}\right)^{2} \frac{\mu_{p}}{(p+1) !}\left(\frac{\pi^{2}}{6}-8 \ln (2)^{2}\right) \epsilon^{a_{0} \cdots a_{p}} H_{i a_{0} \cdots a_{p}} \partial^{a} \partial_{a}\left[\left(\phi^{(1)}-\phi^{(2)}\right)^{i} T T^{*}\right]
$$

One important point has to be made. This coupling and in particular the difference between the first scalar (which is on the brane) and the second scalar (which is on the anti brane) inside this new coupling (35) has to appear, to be able to produce the third term of the expansion in (27), otherwise we get inconsistent result.

Likewise the last section, we are going to find out the closed form of the expansion of $L_{2}$ around (11) as

$$
\begin{aligned}
L_{2}= & \frac{\sqrt{\pi}}{2}\left(\frac{-1}{\left(t^{\prime}+s^{\prime}+u\right)}+\sum_{n=0}^{\infty} a_{n}\left(s^{\prime}+t^{\prime}+u\right)^{n}+\frac{\sum_{n, m=0}^{\infty} l_{n, m}\left(s^{\prime}+t^{\prime}\right)^{n}\left(t^{\prime} s^{\prime}\right)^{m+1}}{\left(t^{\prime}+s^{\prime}+u\right)}\right. \\
& \left.+\sum_{p, n, m=0}^{\infty} e_{p, n, m}\left(s^{\prime}+t^{\prime}+u\right)^{p}\left(s^{\prime}+t^{\prime}\right)^{n}\left(t^{\prime} s^{\prime}\right)^{m+1}\right)
\end{aligned}
$$

$l_{n, m}$ and $e_{p, n, m}$ are

$$
\begin{aligned}
l_{0,0}=-\pi^{2} / 3, & l_{1,0}=8 \zeta(3) \\
l_{2,0}=-7 \pi^{4} / 45, l_{0,1}=\pi^{4} / 45, & l_{3,0}=32 \zeta(5), l_{1,1}=-32 \zeta(5)+8 \zeta(3) \pi^{2} / 3 \\
e_{0,0,0}=\frac{2}{3}\left(2 \pi^{2} \ln (2)-21 \zeta(3)\right), & e_{1,0,0}=\frac{1}{9}\left(4 \pi^{4}-504 \zeta(3) \ln (2)+24 \pi^{2} \ln (2)^{2}\right)
\end{aligned}
$$

It is highly important to trust this special expansion in order to distinguish the last contact terms of (36) from the second terms of expansion in (36). Indeed the last terms in (36) do have different structure so we come to the fact that the last term of the expansion must be produced by making use of the different couplings in field theory.

Using the arguments we have mentioned earlier, we can write down a higher derivative coupling to produce the second term in (36). In the other words it is produced by taking this higher extension of the coupling 


$$
-\left(\alpha^{\prime}\right)^{2} \frac{\mu_{p}}{(p+1) !} \sum_{n=0}^{\infty} a_{n}\left(\frac{\alpha^{\prime}}{2}\right)^{n} \epsilon^{a_{0} \cdots a_{p}} H_{i a_{0} \cdots a_{p}}\left(\partial^{a} \partial_{a}\right)^{n}\left[\left(\phi^{(1)}-\phi^{(2)}\right)^{i} T T^{*}\right]
$$

Remember that we found these couplings with using the S-matrix of string theory, thus we believe that here all on-shell ambiguities for these new couplings are removed.

As it is clear from the closed form of the expansion of $L_{2}$ in (36), it does involve the infinite scalar poles in $t^{\prime}+s^{\prime}+u$-channel for $p+2=n$ case. Let us write down in a precise way all the infinite scalar poles in string theory once more:

$$
\mathcal{A}^{C \phi T_{2} T_{2}}=\frac{4 i \mu_{p}}{(p+1) !} \epsilon^{a_{0} \cdots a_{p}} H_{i a_{0} \cdots a_{p}}\left(\xi^{i} s^{\prime} t^{\prime}\right) \frac{\sum_{n, m=0}^{\infty} l_{n, m}\left(s^{\prime}+t^{\prime}\right)^{n}\left(t^{\prime} s^{\prime}\right)^{m}}{\left(t^{\prime}+s^{\prime}+u\right)}
$$

In this subsection, we want to obviously show that the recent all order two scalar two tachyon couplings of non-BPS branes (equation (43) of [18]) will not produce infinite massless scalar poles of brane anti brane 's string theory amplitude of $C \phi T_{2} T_{2}$ which exist in $\left(s^{\prime}+t^{\prime}+u\right)$-channel.

Thus we conclude that, one has to discover the new all order higher derivative corrections of two tachyon, two scalar couplings of brane anti brane systems. Finally in order to show that we have found the correct corrections to two tachyon two scalar couplings to all orders in brane anti brane systems we make a consistent check by producing all infinite scalar poles of our S-Matrix $(C \phi T T)$.

In fact by making use of these new higher derivative corrections we exactly produce all the infinite scalar poles in $u+t^{\prime}+s^{\prime}$ channel. Although we do not explain all details for corrections, we just make a comment that the appearance of $\sigma_{3}$ Chan-Paton factor for scalar field in (-1)-picture plays a crucial role to actually obtain these corrections (more information can be found in [18, 35]).

The Lagrangian for two scalar, two tachyon couplings for non-BPS brane was defined as

$$
\begin{aligned}
\mathcal{L}(\phi, \phi, T, T)= & -2 T_{p}\left(\pi \alpha^{\prime}\right)^{3} \operatorname{STr}\left(m^{2} T^{2}\left(D_{a} \phi^{i} D^{a} \phi_{i}\right)+\frac{\alpha^{\prime}}{2} D^{\alpha} T D_{\alpha} T D_{a} \phi^{i} D^{a} \phi_{i}\right. \\
& \left.-\alpha^{\prime} D^{b} T D^{a} T D_{a} \phi^{i} D_{b} \phi_{i}\right)
\end{aligned}
$$

Having extracted symmetrized traces, the higher derivative corrections of two scalars, two tachyons for non-BPS branes to all orders in $\alpha^{\prime}$ have been found in [18] as 


$$
\mathcal{L}=-2 T_{p}\left(\pi \alpha^{\prime}\right)\left(\alpha^{\prime}\right)^{2+n+m} \sum_{n, m=0}^{\infty}\left(\mathcal{L}_{1}^{n m}+\mathcal{L}_{2}^{n m}+\mathcal{L}_{3}^{n m}+\mathcal{L}_{4}^{n m}\right)
$$

where

$$
\begin{aligned}
\mathcal{L}_{1}^{n m}= & m^{2} \operatorname{Tr}\left(a_{n, m}\left[\mathcal{D}_{n m}\left(T^{2} D_{a} \phi^{i} D^{a} \phi_{i}\right)+\mathcal{D}_{n m}\left(D_{a} \phi^{i} D^{a} \phi_{i} T^{2}\right)\right]\right. \\
& \left.+b_{n, m}\left[\mathcal{D}_{n m}^{\prime}\left(T D_{a} \phi^{i} T D^{a} \phi_{i}\right)+\mathcal{D}_{n m}^{\prime}\left(D_{a} \phi^{i} T D^{a} \phi_{i} T\right)\right]+h . c .\right) \\
\mathcal{L}_{2}^{n m}= & \operatorname{Tr}\left(a_{n, m}\left[\mathcal{D}_{n m}\left(D^{\alpha} T D_{\alpha} T D_{a} \phi^{i} D^{a} \phi_{i}\right)+\mathcal{D}_{n m}\left(D_{a} \phi^{i} D^{a} \phi_{i} D^{\alpha} T D_{\alpha} T\right)\right]\right. \\
& \left.+b_{n, m}\left[\mathcal{D}_{n m}^{\prime}\left(D^{\alpha} T D_{a} \phi^{i} D_{\alpha} T D^{a} \phi_{i}\right)+\mathcal{D}_{n m}^{\prime}\left(D_{a} \phi^{i} D_{\alpha} T D^{a} \phi_{i} D^{\alpha} T\right)\right]+h . c .\right) \\
\mathcal{L}_{3}^{n m}= & -\operatorname{Tr}\left(a_{n, m}\left[\mathcal{D}_{n m}\left(D^{\beta} T D_{\mu} T D^{\mu} \phi^{i} D_{\beta} \phi_{i}\right)+\mathcal{D}_{n m}\left(D^{\mu} \phi^{i} D_{\beta} \phi_{i} D^{\beta} T D_{\mu} T\right)\right]\right. \\
& \left.+b_{n, m}\left[\mathcal{D}_{n m}^{\prime}\left(D^{\beta} T D^{\mu} \phi^{i} D_{\mu} T D_{\beta} \phi_{i}\right)+\mathcal{D}_{n m}^{\prime}\left(D^{\mu} \phi^{i} D_{\mu} T D_{\beta} \phi_{i} D^{\beta} T\right)\right]+h . c .\right) \\
\mathcal{L}_{4}^{n m}= & -\operatorname{Tr}\left(a_{n, m}\left[\mathcal{D}_{n m}\left(D^{\beta} T D^{\mu} T D_{\beta} \phi^{i} D_{\mu} \phi_{i}\right)+\mathcal{D}_{n m}\left(D^{\beta} \phi^{i} D^{\mu} \phi_{i} D_{\beta} T D_{\mu} T\right)\right]\right. \\
& \left.+b_{n, m}\left[\mathcal{D}_{n m}^{\prime}\left(D^{\beta} T D_{\beta} \phi^{i} D^{\mu} T D_{\mu} \phi_{i}\right)+\mathcal{D}_{n m}^{\prime}\left(D_{\beta} \phi^{i} D_{\mu} T D^{\mu} \phi_{i} D^{\beta} T\right)\right]+h . c .\right)
\end{aligned}
$$

Note that the definitions of $\mathcal{D}_{n m}(E F G H)$ and $\mathcal{D}_{n m}^{\prime}(E F G H)$ were given in [18]. Let us show in a clear way that these corrections do not work for brane anti brane systems, namely we want to emphasize that by using them we can not produce all the infinite scalar poles in $u+t^{\prime}+s^{\prime}$-channel. Thus it definitely means that they are not correct corrections for brane anti brane systems and one has to discover new corrections for two tachyons and two scalars of brane anti brane system and distinguish them from the corrections of two tachyons and two scalars of non-BPS branes, which is one of the main goals of this paper.

The Feynman rule for this particular case is

$$
\mathcal{A}=V_{\alpha}^{i}\left(C_{p+1}, \phi\right) G_{\alpha \beta}^{i j}(\phi) V_{\beta}^{j}\left(\phi, \phi_{1}, T_{2}, T_{2}\right)
$$

with

$$
\begin{aligned}
G_{\alpha \beta}^{i j}(\phi) & =\frac{-i \delta_{\alpha \beta} \delta^{i j}}{T_{p}\left(2 \pi \alpha^{\prime}\right)^{2} k^{2}}=\frac{-i \delta_{\alpha \beta} \delta^{i j}}{T_{p}\left(2 \pi \alpha^{\prime}\right)^{2}\left(t^{\prime}+s^{\prime}+u\right)}, \\
V_{\alpha}^{i}\left(C_{p+1}, \phi\right) & =i\left(2 \pi \alpha^{\prime}\right) \mu_{p} \frac{1}{(p+1) !} \epsilon^{a_{0} \cdots a_{p}} H_{a_{0} \cdots a_{p}}^{i} \operatorname{Tr}\left(\lambda_{\alpha}\right) .
\end{aligned}
$$


$\lambda_{\alpha}$ is an Abelian matrix. Having considered off-shell 's scalar field which is Abelian and taking two permutations as

$$
\operatorname{Tr}\left(\lambda_{2} \lambda_{3} \lambda_{1} \lambda_{\beta}\right), \operatorname{Tr}\left(\lambda_{2} \lambda_{3} \lambda_{\beta} \lambda_{1}\right)
$$

$V_{\beta}^{j}\left(\phi, \phi_{1}, T_{2}, T_{2}\right)$ should be derived from the higher derivative couplings in (42) as

$$
\begin{aligned}
V_{\beta}^{j}\left(\phi, \phi_{1}, T_{2}, T_{2}\right)= & \xi_{1}^{j} I_{9}\left(-2 i T_{p} \pi\right)\left(\alpha^{\prime}\right)^{n+m+3}\left(a_{n, m}+b_{n, m}\right)\left(\left(k_{2} \cdot k_{1}\right)^{n}\left(k \cdot k_{2}\right)^{m}+\left(k_{2} \cdot k_{1}\right)^{n}\left(k_{3} \cdot k_{1}\right)^{m}\right. \\
& +\left(k_{2} \cdot k\right)^{n}\left(k_{1} \cdot k_{2}\right)^{m}+\left(k \cdot k_{2}\right)^{n}\left(k \cdot k_{3}\right)^{m}+\left(k_{3} \cdot k\right)^{n}\left(k_{2} \cdot k\right)^{m}+\left(k_{3} \cdot k\right)^{n}\left(k_{3} \cdot k_{1}\right)^{m} \\
& \left.+\left(k_{1} \cdot k_{3}\right)^{n}\left(k_{2} \cdot k_{1}\right)^{m}+\left(k_{3} \cdot k_{1}\right)^{n}\left(k_{3} \cdot k\right)^{m}\right)
\end{aligned}
$$

like previous notations $k$ becomes off-shell scalar field's momentum, and

$$
I_{9}=\operatorname{Tr}\left(\lambda_{1} \lambda_{2} \lambda_{3} \lambda_{\beta}\right) \frac{1}{2}\left(s^{\prime}\right)\left(t^{\prime}\right)
$$

$b_{n, m}$ is symmetric, see [6]. Although the coefficients have been mentioned in [18], listing some of the coefficients like $a_{n, m}$ and $b_{n, m}$ is important to address :

$$
\begin{aligned}
& a_{0,0}=-\frac{\pi^{2}}{6}, b_{0,0}=-\frac{\pi^{2}}{12}, a_{1,0}=2 \zeta(3), a_{0,1}=0, b_{0,1}=-\zeta(3), a_{1,1}=a_{0,2}=-7 \pi^{4} / 90, \\
& a_{2,2}=\left(-83 \pi^{6}-7560 \zeta(3)^{2}\right) / 945, b_{2,2}=-\left(23 \pi^{6}-15120 \zeta(3)^{2}\right) / 1890, a_{1,3}=-62 \pi^{6} / 945, \\
& a_{2,0}=-4 \pi^{4} / 90, b_{1,1}=-\pi^{4} / 180, b_{0,2}=-\pi^{4} / 45, a_{0,4}=-31 \pi^{6} / 945, a_{4,0}=-16 \pi^{6} / 945, \\
& a_{1,2}=a_{2,1}=8 \zeta(5)+4 \pi^{2} \zeta(3) / 3, a_{0,3}=0, a_{3,0}=8 \zeta(5), b_{1,3}=-\left(12 \pi^{6}-7560 \zeta(3)^{2}\right) / 1890, \\
& a_{3,1}=\left(-52 \pi^{6}-7560 \zeta(3)^{2}\right) / 945, b_{0,3}=-4 \zeta(5), b_{1,2}=-8 \zeta(5)+2 \pi^{2} \zeta(3) / 3, \\
& b_{0,4}=-16 \pi^{6} / 1890 .
\end{aligned}
$$

One needs to use the following relations as well

$$
k_{3} \cdot k=k_{2} \cdot k_{1}-k^{2}, \quad k_{2} \cdot k=k_{1} \cdot k_{3}-k^{2}
$$

By ignoring some of the contact interactions (we go through them in the next section), we get all poles in field theory as

$$
8 i \mu_{p} \frac{\epsilon^{a_{0} \cdots a_{p}} \xi_{i} H_{a_{0} \cdots a_{p}}^{i}}{(p+1) !\left(s^{\prime}+t^{\prime}+u\right)} \operatorname{Tr}\left(\lambda_{1} \lambda_{2} \lambda_{3}\right) \sum_{n, m=0}^{\infty}\left(a_{n, m}+b_{n, m}\right)\left[s^{\prime m} t^{\prime n}+s^{\prime n} t^{\prime m}\right] s^{\prime} t^{\prime}
$$

On the other hand all the infinite scalar poles of the amplitude in string theory are given as 


$$
\mathcal{A}^{\phi_{1} T_{2} T_{2} C}= \pm \frac{4 i \mu_{p}}{(p+1) !} \epsilon^{a_{0} \cdots a_{p}} H_{a_{0} \cdots a_{p}}^{i} \frac{\sum_{n, m=0}^{\infty} l_{n, m}\left(s^{\prime}+t^{\prime}\right)^{n}\left(t^{\prime} s^{\prime}\right)^{m+1}}{\left(t^{\prime}+s^{\prime}+u\right)} \xi_{i}
$$

Now we explicitly show that the corrections of non-BPS branes in (42) can not be used for brane anti brane systems. After removing the common coefficients from both string and field theory amplitude, we are going to compare both string and field theory amplitude at zero and first order of $\alpha^{\prime}$ such that at zero order in field theory we get

$$
16 s^{\prime} t^{\prime}\left(a_{0,0}+b_{0,0}\right)=-4 \pi^{2} s^{\prime} t^{\prime}
$$

while in string theory we get

$$
4 l_{0,0} s^{\prime} t^{\prime}=\frac{-4 \pi^{2}}{3} s^{\prime} t^{\prime}=4 l_{0,0} s^{\prime} t^{\prime}
$$

Since these two coefficients are not the same, clearly it shows that we can not make those corrections of non-BPS branes (42) to brane anti brane systems. Let us also see what happens at first order of $\alpha^{\prime}$ in both string and field theory sides.

At $\alpha^{\prime}$ order, the field theory amplitude has

$$
8 s^{\prime} t^{\prime}\left(s^{\prime}+t^{\prime}\right)\left(a_{1,0}+a_{0,1}+b_{0,1}+b_{0,1}\right)=0
$$

while in string theory at first order we get

$$
4 l_{1,0} s^{\prime} t^{\prime}\left(s^{\prime}+t^{\prime}\right)=32 \zeta(3) s^{\prime} t^{\prime}\left(s^{\prime}+t^{\prime}\right)
$$

Thus even at first order of $\alpha^{\prime}$ it certainly confirms that (42) gives us the wrong result for brane anti brane systems. Therefore we come to important fact that one has to find out the correct higher derivative corrections of brane anti brane systems to all orders of $\alpha^{\prime}$ in order to be able to produce all infinite massless scalar poles in $t^{\prime}+s^{\prime}+u$ - channel. In the next section we construct them and check them out for all orders of $\alpha^{\prime}$.

\subsection{Higher derivative corrections to two tachyon-two scalar cou- plings for brane anti brane systems to all orders in $\alpha^{\prime}$}

In this section we are going to propose higher derivative corrections to two tachyons, two scalar field couplings in brane anti brane systems to all orders in $\alpha^{\prime}$. As it is obvious the 
action for brane anti brane system might be constructed by making use of the projection on the effective actions of two unstable branes. Indeed the projection is $(-1)^{F_{L}}$ where $F_{L}$ becomes space time 's left handed fermion number. Details for constructing higher derivative corrections to all orders in $\alpha^{\prime}$, for BPS branes in [6, 34], for non-BPS branes in [18, 17, 35] and for brane anti brane systems namely for the couplings between two tachyons and two gauge fields in [19] were given.

In order to avoid some details we just make a very important point as follows. To derive all the higher derivative corrections it turns out that the internal degree of freedom of the scalar field in (-1)-picture (which is $\sigma_{3}$ ) plays the crucial role.

By substituting the correct Chan-Paton matrices and extracting the related traces (for more information see [35]), we end up indeed with two scalar field and two tachyon couplings to all orders of $\alpha^{\prime}$ for brane anti brane system as below:

$$
\mathcal{L}=-2 T_{p}\left(\pi \alpha^{\prime}\right)\left(\alpha^{\prime}\right)^{2+n+m} \sum_{n, m=0}^{\infty}\left(\mathcal{L}_{1}^{n m}+\mathcal{L}_{2}^{n m}+\mathcal{L}_{3}^{n m}+\mathcal{L}_{4}^{n m}\right)
$$

where

$$
\begin{aligned}
\mathcal{L}_{1}^{n m}= & m^{2} \operatorname{Tr}\left(a_{n, m}\left[\mathcal{D}_{n m}\left(T T^{*} D_{a} \phi^{(1) i} D^{a} \phi_{i}^{(1)}\right)+\mathcal{D}_{n m}\left(D_{a} \phi^{(1) i} D^{a} \phi_{i}^{(1)} T T^{*}\right)+\text { h.c. }\right]\right. \\
& \left.-b_{n, m}\left[\mathcal{D}_{n m}^{\prime}\left(T D_{a} \phi^{(2) i} T^{*} D^{a} \phi_{i}^{(1)}\right)+\mathcal{D}_{n m}^{\prime}\left(D_{a} \phi^{(1) i} T D^{a} \phi_{i}^{(2)} T^{*}\right)+h . c .\right]\right), \\
\mathcal{L}_{2}^{n m}= & \operatorname{Tr}\left(a_{n, m}\left[\mathcal{D}_{n m}\left(D^{\alpha} T D_{\alpha} T^{*} D_{a} \phi^{(1) i} D^{a} \phi_{i}^{(1)}\right)+\mathcal{D}_{n m}\left(D_{a} \phi^{(1) i} D^{a} \phi_{i}^{(1)} D^{\alpha} T D_{\alpha} T^{*}\right)+\text { h.c. }\right]\right. \\
& \left.-b_{n, m}\left[\mathcal{D}_{n m}^{\prime}\left(D^{\alpha} T D_{a} \phi^{(2) i} D_{\alpha} T^{*} D^{a} \phi_{i}^{(1)}\right)+\mathcal{D}_{n m}^{\prime}\left(D_{a} \phi^{(1) i} D_{\alpha} T D_{a} \phi_{i}^{(2)} D^{\alpha} T^{*}\right)+\text { h.c. }\right]\right), \\
\mathcal{L}_{3}^{n m}= & -\operatorname{Tr}\left(a_{n, m}\left[\mathcal{D}_{n m}\left(D^{\beta} T D_{\mu} T^{*} D^{\mu} \phi^{(1) i} D_{\beta} \phi_{i}^{(1)}\right)+\mathcal{D}_{n m}\left(D^{\mu} \phi^{(1) i} D_{\beta} \phi_{i}^{(1)} D^{\beta} T D_{\mu} T^{*}\right)+\text { h.c. }\right]\right. \\
& \left.-b_{n, m}\left[\mathcal{D}_{n m}^{\prime}\left(D^{\beta} T D^{\mu} \phi^{(2) i} D_{\mu} T^{*} D_{\beta} \phi_{i}^{(1)}\right)+\mathcal{D}_{n m}^{\prime}\left(D^{\mu} \phi^{(1) i} D_{\mu} T D_{\beta} \phi_{i}^{(2)} D^{\beta} T^{*}\right)+\text { h.c. }\right]\right), \\
\mathcal{L}_{4}^{n m}= & -\operatorname{Tr}\left(a_{n, m}\left[\mathcal{D}_{n m}\left(D^{\beta} T D^{\mu} T^{*} D_{\beta} \phi^{(1) i} D_{\mu} \phi_{i}^{(1)}\right)+\mathcal{D}_{n m}\left(D^{\beta} \phi^{(1) i} D^{\mu} \phi_{i}^{(1)} D_{\beta} T D_{\mu} T^{*}\right)+\text { h.c. }\right]\right. \\
& \left.-b_{n, m}\left[\mathcal{D}_{n m}^{\prime}\left(D^{\beta} T D_{\beta} \phi^{(2) i} D^{\mu} T^{*} D_{\mu} \phi_{i}^{(1)}\right)+\mathcal{D}_{n m}^{\prime}\left(D_{\beta} \phi^{(1) i} D_{\mu} T D^{\mu} \phi_{i}^{(2)} D^{\beta} T^{*}\right)+\text { h.c. }\right]\right)(52)
\end{aligned}
$$

In addition to the above couplings, one has to interchange $D \phi^{(1) i}$ to $D \phi^{(2) i}$ just for all the terms involving $a_{n, m}$ and also interchange $D \phi^{(1) i} \leftrightarrow D \phi^{(2) i}$ for all $b_{n, m}$ ' terms and essentially add these terms to above couplings as well.

The other point should be clarified is that here all scalar field's covariant derivatives are indeed partial derivatives. Notice that when fields change very slowly then above couplings 
go back to two scalar, two tachyon couplings of tachyon DBI action. Although here we do not need to actually include gauge fields, however, it is worth trying to point out that

$$
D_{a_{1}} \cdots D_{a_{n}} T=\partial_{a_{1}} D_{a_{2}} \cdots D_{a_{n}} T-i\left(A_{a_{1}}^{(1)}-A_{a_{1}}^{(2)}\right) D_{a_{2}} \cdots D_{a_{n}} T
$$

It is highly recommended to mention once more that in this paper we discovered some new couplings. In particular likewise the first part of the amplitude to make sense of consistent result for brane anti brane system, all the couplings

$$
D \phi^{(1) i} \cdot D \phi_{i}^{(2)}
$$

and their new corrections must be appeared in brane anti brane effective action.

Notice that these new corrections for brane anti brane systems (52) ( all order two tachyon two scalar couplings) can not be derived from the corrections of two tachyon and two gauge fields of the same system (compare equation (46) of [?] with (52)). The reasons are as follows. First of all by direct computations we showed that the sign of all the coefficients of $b_{n, m}$ must get reversed and second of all while the coefficients of $L_{1}, L_{2}$ in both cases are the same, the coefficients of $L_{3}, L_{4}$ are completely different (which are not over all coefficients), that is why we claim the direct computations are needed. This clearly shows that by T-duality transformation, one can guess the general structure of higher derivative corrections but all their coefficients can just be fixed by direct Scattering computations as has been shown and argued in [18, 5, 34.

Now in order to show that we have obtained consistent higher derivative corrections of brane anti brane to all orders in $\alpha^{\prime}$, we would like to use these new couplings in (52) to indeed check out all infinite massless scalar poles of our amplitude $C \phi T_{2} T_{2}$. This is a very important check to do so.

Therefore consider one $\mathrm{RR}-(p+1)$ and one scalar and two either $T_{1}$ or $T_{2}$ tachyons for brane anti brane system, the Feynman rule in (42) must be taken as well.

Here as we have mentioned earlier on (at the moment) we ignore some contact interactions and just focus on new higher derivative corrections of two scalars two tachyons of brane anti brane to be able to produce all the infinite scalar poles.

Now making use of the new two scalar two tachyon couplings of (52) and taking two possible permutations,$V_{\beta}^{j}\left(\phi, \phi_{1}, T_{2}, T_{2}\right)$ might be found as 


$$
\begin{aligned}
V_{\beta}^{j}\left(\phi_{1}, \phi_{1}, T_{2}, T_{2}\right)= & \xi_{1}^{j}\left(-2 i T_{p} \pi\right)\left(\alpha^{\prime}\right)^{n+m+3}\left(a_{n, m}-b_{n, m}\right)\left(\left(k_{2} \cdot k_{1}\right)^{n}\left(k \cdot k_{2}\right)^{m}+\left(k_{2} \cdot k_{1}\right)^{n}\left(k_{3} \cdot k_{1}\right)^{m}\right. \\
& +\left(k_{2} \cdot k\right)^{n}\left(k_{1} \cdot k_{2}\right)^{m}+\left(k \cdot k_{2}\right)^{n}\left(k \cdot k_{3}\right)^{m}+\left(k_{3} \cdot k\right)^{n}\left(k_{2} \cdot k\right)^{m}+\left(k_{3} \cdot k\right)^{n}\left(k_{3} \cdot k_{1}\right)^{m} \\
& \left.+\left(k_{1} \cdot k_{3}\right)^{n}\left(k_{2} \cdot k_{1}\right)^{m}+\left(k_{3} \cdot k_{1}\right)^{n}\left(k_{3} \cdot k\right)^{m}\right) \frac{1}{2}\left(s^{\prime}\right)\left(t^{\prime}\right) \operatorname{Tr}\left(\lambda_{1} \lambda_{2} \lambda_{3} \lambda_{\beta}\right)
\end{aligned}
$$

Note that $\phi$ in the propagator of $G_{i j}(\phi)$ must be $\phi^{(1)}, \phi^{(2)}$. Let us highlight that $T_{2}$ is related to the second component of complex scalar tachyon which we pointed out earlier. Considering the following vertices

$$
\begin{aligned}
G_{i j}(\phi)= & \frac{i \delta_{i j} \delta_{\alpha \beta}}{\left(2 \pi \alpha^{\prime}\right)^{2} T_{p}\left(u+t^{\prime}+s^{\prime}\right)} \\
V_{i}\left(C_{p+1}, \phi^{(1)}\right)= & i \mu_{p}\left(2 \pi \alpha^{\prime}\right) \frac{1}{(p+1) !} \epsilon^{a_{0} \cdots a_{p}} H_{i a_{0} \cdots a_{p}} \operatorname{Tr}\left(\lambda_{\alpha}\right) \\
V_{i}\left(C_{p+1}, \phi^{(2)}\right)= & -i \mu_{p}\left(2 \pi \alpha^{\prime}\right) \frac{1}{(p+1) !} \epsilon^{a_{0} \cdots a_{p}} H_{i a_{0} \cdots a_{p}} \operatorname{Tr}\left(\lambda_{\alpha}\right) \\
V_{\beta}^{j}\left(\phi_{2}, \phi_{1}, T_{2}, T_{2}\right)= & \xi_{1}^{j}\left(2 i T_{p} \pi\right)\left(\alpha^{\prime}\right)^{n+m+3}\left(a_{n, m}-b_{n, m}\right)\left(\left(k_{2} \cdot k_{1}\right)^{n}\left(k \cdot k_{2}\right)^{m}+\left(k_{2} \cdot k_{1}\right)^{n}\left(k_{3} \cdot k_{1}\right)^{m}\right. \\
& +\left(k_{2} \cdot k\right)^{n}\left(k_{1} \cdot k_{2}\right)^{m}+\left(k \cdot k_{2}\right)^{n}\left(k \cdot k_{3}\right)^{m}+\left(k_{3} \cdot k\right)^{n}\left(k_{2} \cdot k\right)^{m}+\left(k_{3} \cdot k\right)^{n}\left(k_{3} \cdot k_{1}\right)^{m} \\
& \left.+\left(k_{1} \cdot k_{3}\right)^{n}\left(k_{2} \cdot k_{1}\right)^{m}+\left(k_{3} \cdot k_{1}\right)^{n}\left(k_{3} \cdot k\right)^{m}\right) \frac{1}{2}\left(s^{\prime}\right)\left(t^{\prime}\right) \operatorname{Tr}\left(\lambda_{1} \lambda_{2} \lambda_{3} \lambda_{\beta}\right),
\end{aligned}
$$

and implementing (54) into (42) we get all infinite scalar poles in field theory as

$$
8 i \mu_{p} \frac{\epsilon^{a_{0} \cdots a_{p}} \xi_{i} H_{a_{0} \cdots a_{p}}^{i}}{(p+1) !\left(s^{\prime}+t^{\prime}+u\right)} \operatorname{Tr}\left(\lambda_{1} \lambda_{2} \lambda_{3}\right) \sum_{n, m=0}^{\infty}\left(a_{n, m}-b_{n, m}\right)\left[s^{\prime m} t^{\prime n}+s^{\prime n} t^{\prime m}\right] s^{\prime} t^{\prime}
$$

Simultaneously all the infinite scalar poles of the string amplitude are

$$
\mathcal{A}^{\phi_{1} T_{1} T_{1} C}= \pm \frac{4 i \mu_{p}}{(p+1) !} \epsilon^{a_{0} \cdots a_{p}} H_{a_{0} \cdots a_{p}}^{i} \frac{\sum_{n, m=0}^{\infty} l_{n, m}\left(s^{\prime}+t^{\prime}\right)^{n}\left(t^{\prime} s^{\prime}\right)^{m+1}}{\left(t^{\prime}+s^{\prime}+u\right)} \xi_{i}
$$

Now if we have discovered (52) correctly, we would have to exactly produce all the infinite scalar poles of string theory in field theory side as well.

Let us remove common factors and just compare string poles with field theory poles at each order of $\alpha^{\prime}$. 
By setting $n=m=0$, the string amplitude gives rise $4 l_{0,0} s^{\prime} t^{\prime}$ coefficient as it is shown in (50). At the same time at zeroth order of $\alpha^{\prime}$ the field theory amplitude gives us

$$
16 s^{\prime} t^{\prime}\left(a_{0,0}-b_{0,0}\right)=-4 \frac{\pi^{2}}{3} s^{\prime} t^{\prime}
$$

which is precisely equivalent to $4 l_{0,0} s^{\prime} t^{\prime}$ (the same coefficient in string side).

Now we can see that the new two scalar, two tachyon couplings of brane anti brane (52) work out. In $\alpha^{\prime}$ order, from string's amplitude we obtain

$$
4 l_{1,0} s^{\prime} t^{\prime}\left(s^{\prime}+t^{\prime}\right)
$$

and in particular in field 's amplitude we get to

$$
8 s^{\prime} t^{\prime}\left(s^{\prime}+t^{\prime}\right)\left(a_{1,0}+a_{0,1}-b_{0,1}-b_{0,1}\right)=32 \zeta(3)\left(s^{\prime}+t^{\prime}\right) s^{\prime} t^{\prime}=4 l_{1,0} s^{\prime} t^{\prime}\left(s^{\prime}+t^{\prime}\right)
$$

For $\left(\alpha^{\prime}\right)^{2}$ order, string amplitude related to

$$
4 l_{2,0} s^{\prime} t^{\prime}\left(s^{\prime}+t^{\prime}\right)^{2}+4 l_{0,1}\left(s^{\prime} t^{\prime}\right)^{2}
$$

and field theory amplitude carries

$$
\begin{aligned}
& 16\left(s^{\prime} t^{\prime}\right)^{2}\left(a_{1,1}-b_{1,1}\right)+8 s^{\prime} t^{\prime}\left(a_{0,2}+a_{2,0}-b_{0,2}-b_{2,0}\right)\left[\left(s^{\prime}\right)^{2}+\left(t^{\prime}\right)^{2}\right] \\
& =4 s^{\prime} t^{\prime}\left(-\frac{7 \pi^{4}}{45}\left(s^{\prime}+t^{\prime}\right)^{2}+\frac{\pi^{4}}{45} s^{\prime} t^{\prime}\right)=4\left(l_{2,0}\left(s^{\prime}+t^{\prime}\right)^{2}+l_{0,1} s^{\prime} t^{\prime}\right) s^{\prime} t^{\prime}
\end{aligned}
$$

Finally we would like to check $\alpha^{\prime 3}$ order, that is, string amplitude does carry

$$
4 l_{3,0}\left(s^{\prime}+t^{\prime}\right)^{3} t^{\prime} s^{\prime}+4 l_{1,1}\left(s^{\prime}+t^{\prime}\right)\left(s^{\prime} t^{\prime}\right)^{2}
$$

and field amplitude does include

$$
\begin{aligned}
& 8 s^{\prime} t^{\prime}\left(a_{3,0}+a_{0,3}-b_{0,3}-b_{3,0}\right)\left[\left(s^{\prime}\right)^{3}+\left(t^{\prime}\right)^{3}\right]+8 s^{\prime} t^{\prime}\left(a_{1,2}+a_{2,1}-b_{1,2}-b_{2,1}\right) s^{\prime} t^{\prime}\left(s^{\prime}+t^{\prime}\right) \\
& =128 \zeta(5) s^{\prime} t^{\prime}\left(s^{\prime 3}+t^{\prime 3}\right)+8 s^{\prime 2} t^{\prime 2}\left(s^{\prime}+t^{\prime}\right)\left(32 \zeta(5)+4 \pi^{2} \zeta(3) / 3\right)
\end{aligned}
$$

which is exactly equivalent to the same coefficients $4 l_{3,0}\left(s^{\prime}+t^{\prime}\right)^{3} t^{\prime} s^{\prime}+4 l_{1,1}\left(s^{\prime}+t^{\prime}\right)\left(s^{\prime} t^{\prime}\right)^{2}$ in string side.

We can easily do check all the corrections up to all orders, so we reach to the important point that, we have exactly explored all order two tachyon, two scalar couplings of brane anti brane systems. 
Remember in order to get consistent result, some new coupling like the multiplication of $D \phi^{(1) i}$ and $D \phi_{i}^{(2)}$, that is,

$$
D \phi^{(1) i} \cdot D \phi_{i}^{(2)}
$$

has to appear inside these infinite higher derivative corrections, this fact becomes obvious if we concentrate on non zero $b_{n, m}$ coefficients in (52).

Let us complete our discussions even for contact interactions which have been overlooked in producing infinite scalar poles for $p=n+2$ case in field theory analysis. They are given by

$$
\begin{aligned}
& -i \mu_{p}\left(\alpha^{\prime}\right)^{2} \frac{\epsilon^{a_{0} \cdots a_{p}} H_{a_{0} \cdots a_{p}}^{i}}{(p+1) !}\left(-\xi_{i}\left(s^{\prime}\right)\left(t^{\prime}\right)\right) \sum_{n, m=0}^{\infty}\left(a_{n, m}-b_{n, m}\right) \\
& {\left[\left(2 \sum_{\ell=1}^{m}\left(\begin{array}{c}
m \\
\ell
\end{array}\right)\left(t^{\prime m-\ell} s^{\prime n}+s^{\prime m-\ell} t^{\prime n}\right)+2 \sum_{\ell=1}^{n}\left(\begin{array}{c}
n \\
\ell
\end{array}\right)\left(t^{\prime n-\ell} s^{\prime m}+s^{\prime n-\ell} t^{\prime m}\right)\right)\left(\alpha^{\prime} k^{2}\right)^{\ell-1}\right.} \\
& \left.+\sum_{\ell=1, j=1}^{n, m}\left(\begin{array}{c}
n \\
\ell
\end{array}\right)\left(\begin{array}{c}
m \\
j
\end{array}\right)\left(t^{\prime n-\ell} s^{\prime m-j}+s^{\prime n-\ell} t^{\prime m-j}\right)\left(\alpha^{\prime} k^{2}\right)^{\ell+j-1}\right]
\end{aligned}
$$

These couplings might be written down as

$$
\begin{aligned}
& i\left(\alpha^{\prime}\right)^{2} \mu_{p} \frac{\epsilon^{a_{0} \cdots a_{p}} H_{a_{0} \cdots a_{p}}^{i}}{(p+1) !}\left[-\xi_{i} s^{\prime} t^{\prime}\right] \\
& \times \sum_{p, n, m=0}^{\infty} e_{p, n, m}^{\prime}\left(s^{\prime}+t^{\prime}+u\right)^{p}\left(s^{\prime}+t^{\prime}\right)^{n}\left(t^{\prime} s^{\prime}\right)^{m}
\end{aligned}
$$

Notice that we can write $e_{p, n, m}^{\prime}$ as $a_{n, m}$ and $b_{n, m}$. Some of the contact interactions for $C \phi T_{1} T_{1}$ may have precise structure like the above couplings so as a matter of fact one has to replace the coefficients $e_{p, n, m}$ in (36) by $e_{p, n, m}-e_{p, n, m}^{\prime}$

Concerning some of the on-shell ambiguities, all we can express is that, one has to carry out a five point open super string computation like TT $\phi \phi A$ to over come some of the ambiguities however, we can not come over to this computation in this paper. This problem would remain open question to the future research projects.

Eventually let us end up this section by producing all infinite contact interactions of the $C \phi T_{1} T_{1}$ amplitude to all orders in $\alpha^{\prime}$. We just need to take into account the last term in (36) Such that, all contact interactions for this part of the amplitude can be summarized as 


$$
4 i \mu_{p} \frac{\epsilon^{a_{0} \cdots a_{p}} H_{i a_{0} \cdots a_{p}}}{(p+1) !}\left[\xi^{i} s^{\prime} t^{\prime}\right] \sum_{p, n, m=0}^{\infty} e_{p, n, m}\left(s^{\prime}+t^{\prime}+u\right)^{p}\left(s^{\prime}+t^{\prime}\right)^{n}\left(\left(t^{\prime}\right)\left(s^{\prime}\right)\right)^{m}
$$

These terms can be reproduced in field theory by taking the following couplings

$$
\begin{aligned}
2\left(\alpha^{\prime}\right)^{2} \mu_{p} \frac{\epsilon^{a_{0} \cdots a_{p}} H_{a_{0} \cdots a_{p}}^{i}}{(p+1) !} \sum_{p, n, m=0}^{\infty} e_{p, n, m}\left(s^{\prime}+t^{\prime}+u\right)^{p}\left(s^{\prime}+t^{\prime}\right)^{n}\left(\left(t^{\prime}\right)\left(s^{\prime}\right)\right)^{m} \\
\times\left[\partial_{b} \partial_{c}\left(\phi_{1}-\phi_{2}\right)_{i} D^{b} T_{1} D^{c} T_{1}+T_{1} \rightarrow T_{2}\right]
\end{aligned}
$$

Note that in the above couplings the commutator in the definition of tachyon's covariant derivative should not be considered as we do not have any external gauge field here.

In order to produce all the infinite contact interactions, the following derivative must act on the above coupling as well .

$$
\begin{aligned}
\left(\left(s^{\prime}\right)\left(t^{\prime}\right)\right)^{m} H \phi T T & \rightarrow\left(\alpha^{\prime}\right)^{2 m} H \partial_{a_{1}} \cdots \partial_{a_{2 m}} \phi D^{a_{1}} \cdots D^{a_{m}} T D^{a_{m+1}} \cdots D^{a_{2 m}} T \\
\left(s^{\prime}+t^{\prime}\right)^{n} H \phi T T & \rightarrow\left(\alpha^{\prime}\right)^{n} H \partial^{a_{1}} \cdots \partial^{a_{n}} \phi D_{a_{1}} \cdots D_{a_{n}}(T T) \\
\left(s^{\prime}+t^{\prime}+u\right)^{p} H \phi T T & \rightarrow\left(\frac{\alpha^{\prime}}{2}\right)^{p} H\left(D_{a} D^{a}\right)^{p}(\phi T T)
\end{aligned}
$$

One has to pay particular attention to the fact that the above couplings indeed have some on-shell ambiguities.

These ambiguities should be fixed by computing the amplitude of $C T T \phi \phi$ in the world volume of brane anti brane systems. It is understood by field theory analysis that above couplings definitely will be appeared in the infinite tachyon poles of $C T T \phi \phi$ amlitude. It would be interesting to carry out this computation in detail.

\section{Conclusions}

In this paper using direct computations, we have obtained the closed form of the amplitude of one RR, two tachyons and one scalar field in the world volume of brane anti brane systems. We discovered that the vertex of two tachyons and one gauge field $V^{a}\left(A, T_{1}, T_{2}\right)$ should not receive any correction and in particular by obtaining the infinite higher derivative corrections of one RR, one scalar and one gauge field of BPS branes, we could explore all the infinite gauge poles of the amplitude of $C \phi T T$ for $p=n$ case. The special expansion (with taking into account the related Feynman rules) dictates us that leading terms do correspond 
to the DBI and Wess-Zumino effective actions, however all the other non leading terms in the expansion should be related to their higher derivative corrections.

In the case of D-brane/anti D-brane the derivative expansion cannot be thought of as a low energy expansion since the mass of the tachyon is of order of the string scale. Nevertheless we argued that a particular kinematic limit can be used to define a related expansion. It is very important to emphasize that we have discovered all the higher derivative corrections to two tachyon, two scalar field couplings in the world volume of brane anti brane systems to all orders in $\alpha^{\prime}$ in (52) and check them out by producing all the infinite scalar $\left(u+s^{\prime}+t^{\prime}\right)$-channel poles of our amplitude for $p+2=n$ case.

To make sense of super string computations, one has to distinguish the new corrections of brane anti brane systems in (52) from non-BPS branes' corrections in (42).

Infinite gauge poles have provided remarkable information to actually derive all infinite higher derivative corrections of $\partial_{i} C_{p-1} \wedge F\left(\phi_{1}+\phi_{2}\right)^{i}$.

By analysing contact interactions of the amplitude for $p=n$ case we obtained a new coupling like $\partial_{i} C_{p-1} \wedge D T \wedge D T^{*}\left(\phi_{1}+\phi_{2}\right)^{i}$ and fixed its coefficient, we also found all its infinite extensions.

Note that, there is no correction to $\operatorname{Tr}\left(\phi^{i}\right) H_{i a_{0} \cdots a_{p}} \epsilon^{a_{0} \cdots a_{p}}$, therefore all higher scalar poles have provided the complete information for infinite higher derivative corrections to two scalars, two tachyons of brane anti brane systems. Contact interactions for $p+2=n$ give us remarkable clues, not only on new coupling like $\epsilon^{a_{0} \cdots a_{p}} H_{i a_{0} \cdots a_{p}}\left(\phi^{(1)}-\phi^{(2)}\right)^{i} T T^{*}$ but also on infinite higher derivative corrections thereof. We also derived a new coupling like

$$
\begin{aligned}
2\left(\alpha^{\prime}\right)^{2} \mu_{p} \frac{\epsilon^{a_{0} \cdots a_{p}} H_{a_{0} \cdots a_{p}}^{i}}{(p+1) !} \sum_{p, n, m=0}^{\infty} e_{p, n, m}\left(s^{\prime}+t^{\prime}+u\right)^{p}\left(s^{\prime}+t^{\prime}\right)^{n}\left(\left(t^{\prime}\right)\left(s^{\prime}\right)\right)^{m} \\
\times\left[\partial_{b} \partial_{c}\left(\phi_{1}-\phi_{2}\right)_{i} D^{b} T_{1} D^{c} T_{1}+T_{1} \rightarrow T_{2}\right]
\end{aligned}
$$

it would be interesting to see whether or not commutator in the definition of the covariant derivative of tachyon can be held. To settle this remark, one must carry out either $C T T \phi A$ or $C T T \phi A A$ amplitude in the world volume of brane anti brane systems. It is interesting to perform these amplitudes and find out all infinite higher derivative corrections to their effective actions as well. It would be interesting to check CTTTT amplitude to eventually fix symmetrized trace tachyon effective action in the world volume of brane anti brane systems [41]. Finally we point out that it might be interesting to consider the thermodynamical 
aspects of such an action. It has been argued that, at finite temperature such D-brane/ anti D-brane systems can be stable and related to black holes.

\section{Acknowledgments}

I would like to thank A.Sen, Joe.Polchinski, G.Thompson, F.Quevedo, Rob Myers, A.Belavin, N.Lambert, A.Lerda, A.Sagnotti, G.Veneziano, R.K Gupta and in particular K.S.Narain, J.Maldacena and L.Alvarez-Gaume for several valuable discussions. I also thank the referee for providing several fascinating comments/future directions.

\section{References}

[1] J. Polchinski, "Dirichlet Branes and Ramond-Ramond charges," Phys. Rev. Lett. 75, 4724 (1995) hep-th/9510017].

[2] E. Witten, "Bound states of strings and p-branes," Nucl. Phys. B 460, 335 (1996) hep-th/9510135.

[3] J. Polchinski, "Tasi lectures on D-branes," hep-th/9611050.

[4] C. P. Bachas, "Lectures on D-branes," hep-th/9806199.

[5] E. Hatefi, "Shedding light on new Wess-Zumino couplings with their corrections to all orders in alpha-prime," JHEP 1304, 070 (2013) [arXiv:1211.2413 [hep-th]].

[6] E. Hatefi, "On effective actions of BPS branes and their higher derivative corrections," JHEP 1005, 080 (2010) arXiv:1003.0314 [hep-th]].

[7] R. C. Myers, "Dielectric branes," JHEP 9912 (1999) 022 hep-th/9910053].

[8] M. Li, "Boundary states of D-branes and Dy strings," Nucl. Phys. B 460, 351 (1996) hep-th/9510161.

[9] M. R. Douglas, "Branes within branes," In *Cargese 1997, Strings, branes and dualities* 267-275 hep-th/9512077.

[10] M. B. Green, J. A. Harvey and G. W. Moore, "I-brane inflow and anomalous couplings on d-branes," Class. Quant. Grav. 14 (1997) 47 hep-th/9605033.

[11] M. Gutperle and A. Strominger, "Space - like branes," JHEP 0204, 018 (2002) hep-th/0202210. 
[12] A. Sen, "Rolling tachyon," JHEP 0204, 048 (2002) [hep-th/0203211].

[13] A. Sen, "Tachyon matter," JHEP 0207, 065 (2002) [hep-th/0203265].

[14] N. D. Lambert, H. Liu and J. M. Maldacena, "Closed strings from decaying Dbranes," JHEP 0703, 014 (2007) hep-th/0303139.

[15] A. Sen, "Tachyon dynamics in open string theory," Int. J. Mod. Phys. A 20, 5513 (2005) [hep-th/0410103.

[16] A. Sen, "NonBPS states and Branes in string theory," hep-th/9904207.

[17] M. R. Garousi and E. Hatefi, "More on WZ action of non-BPS branes," JHEP 0903 , 008 (2009) arXiv:0812.4216 [hep-th]].

[18] E. Hatefi, "On higher derivative corrections to Wess-Zumino and Tachyonic actions in type II super string theory," Phys. Rev. D 86, 046003 (2012) arXiv:1203.1329 [hep-th]].

[19] M. R. Garousi and E. Hatefi, "On Wess-Zumino terms of Brane-Antibrane systems," Nucl. Phys. B 800 (2008) 502 [arXiv:0710.5875 [hep-th]].

[20] A. Sen, "Dirac-Born-Infeld action on the tachyon kink and vortex," Phys. Rev. D 68, 066008 (2003) [hep-th/0303057].

[21] A. Sen, "Tachyon condensation on the brane anti-brane system," JHEP 9808, 012 (1998) [hep-th/9805170].

[22] C. Kennedy and A. Wilkins, "Ramond-Ramond couplings on Brane - anti-Brane systems," Phys. Lett. B 464, 206 (1999) hep-th/9905195.

[23] A. Sen, "Stable nonBPS states in string theory," JHEP 9806 (1998) 007 hep-th/9803194.

[24] O. Bergman and M. R. Gaberdiel, "Stable nonBPS D particles," Phys. Lett. B 441, 133 (1998) hep-th/9806155 ; A. Sen, "SO(32) spinors of type I and other solitons on brane - anti-brane pair," JHEP 9809, 023 (1998) [hep-th/9808141]; M. Frau, L. Gallot, A. Lerda and P. Strigazzi, "Stable nonBPS D-branes in type I string theory," Nucl. Phys. B 564, 60 (2000) [hep-th/9903123] ; E. Dudas, J. Mourad and A. Sagnotti, "Charged and uncharged D-branes in various string theories," Nucl. Phys. B 620, 109 (2002) hep-th/0107081 ; E. Eyras and S. Panda, "The Spacetime life of a nonBPS D particle," Nucl. Phys. B 584 (2000) 251 hep-th/0003033 
; E. Eyras and S. Panda, "NonBPS branes in a type I orbifold," JHEP 0105, 056 (2001) hep-th/0009224.

[25] E. Witten, "D-branes and K theory," JHEP 9812, 019 (1998) hep-th/9810188].

[26] G. R. Dvali and S. H. H. Tye, "Brane inflation," Phys. Lett. B 450 (1999) 72 hep-ph/9812483.

[27] C. P. Burgess, M. Majumdar, D. Nolte, F. Quevedo, G. Rajesh and R. J. Zhang, "The Inflationary brane anti-brane universe," JHEP 0107, 047 (2001) hep-th/0105204.

[28] D. Choudhury, D. Ghoshal, D. P. Jatkar and S. Panda, "Hybrid inflation and brane - anti-brane system," JCAP 0307, 009 (2003) hep-th/0305104.

[29] S. Kachru, R. Kallosh, A. D. Linde, J. M. Maldacena, L. P. McAllister and S. P. Trivedi, "Towards inflation in string theory," JCAP 0310, 013 (2003) hep-th/0308055.

[30] R. Casero, E. Kiritsis and A. Paredes, "Chiral symmetry breaking as open string tachyon condensation," Nucl. Phys. B 787, 98 (2007) [hep-th/0702155 [HEP-TH]].

[31] A. Dhar and P. Nag, "Sakai-Sugimoto model, Tachyon Condensation and Chiral symmetry Breaking," JHEP 0801, 055 (2008) arXiv:0708.3233 [hep-th]].

[32] P. Kraus and F. Larsen, "Boundary string field theory of the D anti-D system," Phys. Rev. D 63, 106004 (2001) [hep-th/0012198].

[33] G. Roepstorff, "Superconnections and the Higgs field," J. Math. Phys. 40, 2698 (1999) [hep-th/9801040].

[34] E. Hatefi and I. Y. Park, "More on closed string induced higher derivative interactions on D-branes," Phys. Rev. D 85 (2012) 125039 [arXiv:1203.5553 [hep-th]]. ; E. Hatefi, "Three Point Tree Level Amplitude in Superstring Theory," Nucl. Phys. Proc. Suppl. 216 (2011) 234 arXiv:1102.5042 [hep-th]]. ; E. Hatefi, "Closed string Ramond-Ramond proposed higher derivative interactions on fermionic amplitudes in IIB," arXiv:1302.5024 [hep-th].

[35] E. Hatefi and I. Y. Park, "Universality in all-order $\alpha^{\prime}$ corrections to BPS/non-BPS brane world volume theories," Nucl. Phys. B 864 (2012) 640 arXiv:1205.5079 [hepth]]; E. Hatefi, "All order $\alpha^{\prime}$ higher derivative corrections to non-BPS branes of 
type IIB Super string theory," JHEP 1307, 002 (2013) [arXiv:1304.3711 [hep-th]]; E. Hatefi, "Selection rules, RR couplings on non-BPS branes and their all order $\alpha^{\prime}$-corrections in type IIA(B) super string theories," arXiv:1307.3520 [hep-th].

[36] E. Hatefi, A. J. Nurmagambetov and I. Y. Park, "ADM reduction of IIB on $\mathcal{H}^{p, q}$ to dS braneworld," JHEP 1304, 170 (2013) [arXiv:1210.3825 [hep-th]].

[37] E. Hatefi, A. J. Nurmagambetov and I. Y. Park, " $N^{3}$ entropy of $M 5$ branes from dielectric effect," Nucl. Phys. B 866, 58 (2013) arXiv:1204.2711 [hep-th]]. ; E. Hatefi, A. J. Nurmagambetov and I. Y. Park, "Near-Extremal Black-Branes with n*3 Entropy Growth," Int. J. Mod. Phys. A 27, 1250182 (2012) [arXiv:1204.6303 [hep-th]].

[38] S. de Alwis, R. K. Gupta, E. Hatefi and F. Quevedo, "Stability, Tunneling and Flux Changing de Sitter Transitions in the Large Volume String Scenario," arXiv:1308.1222 [hep-th].

[39] H. Liu and J. Michelson, "-trek III: The search for Ramond-Ramond couplings," Nucl. Phys. B 614, 330 (2001) arXiv:hep-th/0107172]; D. Friedan, E. J. Martinec and S. H. Shenker, "Conformal Invariance, Supersymmetry and String Theory," Nucl. Phys. B 271 (1986) 93; V. A. Kostelecky, O. Lechtenfeld, W. Lerche, S. Samuel and S. Watamura, "Conformal Techniques, Bosonization and Tree Level String Amplitudes," Nucl. Phys. B 288, 173 (1987).

[40] A. Fotopoulos, "On (alpha')**2 corrections to the D-brane action for non-geodesic world-volume embeddings," JHEP 0109, 005 (2001) arXiv:hep-th/0104146.

[41] E. Hatefi, "Symmetrized trace D-brane anti D-brane effective actions and all order $\alpha^{\prime}$ corrections to one RR and four tachyon couplings", [work in progress] 